\title{
THE RENAL CLEARANCES OF SUBSTITUTED HIPPURIC ACID DERIVATIVES AND OTHER AROMATIC ACIDS IN DOG AND MAN ${ }^{1}$
}

\author{
By HOMER W. SMITH, NORMA FINKELSTEIN, LUCY ALIMINOSA, \\ BETTY CRAWFORD, AND MARTHA GRABER
}

(From The Department of Physiology, New York University College of Medicine, New York City)

(Received for publication September 29, 1944)

The efficiency with which diodrast. $(3,5$-diiodo-4-pyridone-1-acetic acid) and hippuran (oiodohippuric acid) are excreted by the renal tubules $(1,2)$ raises the question to what extent the organically-bound iodine in these molecules is responsible for the phenomenon of tubular excretion. Since suitable quantitative methods for the determination of unsubstituted compounds vere not available, substituted derivatives of hippuric. acid which could be determined by appropriate coupling reactions were prepared ${ }^{2}$ and studied under conditions permitting the exact comparison of renal clearances with those of diodrast and hippuran.

Methods of comparing clearances. When two substances, both of which are excreted by the tubules, are presented to the tubular excretory mechanism simultaneously, one substance may depress the tubular excretion of the other $(2,3) .^{3}$ Consequently, in the absence of information to

1 Aided by a grant from the Commonwealth Fund. A preliminary report on this work was given before the American Physiological Society in 1941 (22).

$2 \mathrm{We}$ are indebted to Dr. Kenneth C. Blanchard for the synthesis of o- and p-hydroxyhippuric acid, $p$-aminohippuric acid, 2-pyridone-1 acetic acid, p-aminophenaceturic acid, and several compounds not reported in this paper. Subsequently Merck and Company kindly prepared for us m-aminohippuric acid, p-aminomandelic acid, $m$-hydroxyhippuric acid, and additional p-aminohippuric acid. p-Acetaminohippuric acid and cinnamoyl glycine were prepared by Mr. John Mulvaney under Dr. Blanchard's direction. Large quantities of pyrogen-free 20 per cent solution of sodium p-aminohippurate as well as the anhydrous (reagent) free acid for the preparation of standard solutions were supplied through the courtesy of the Medical Research Division of Sharp and Dohme.

8 That hippuric acid is excreted by the tubules was indicated in preliminary experiments by the fact that the intravenous administration of sodium hippurate markedly depresses the phenol red clearance (without affecting the filtration rate) in the dog. the contrary, the comparison of tubular clearances by the conventional simultaneous method is hazardous since, even if the two clearances are identical, it is possible that the clearance of one substance may be depressed by the presence of the other. Although essential in principle, this precaution has proved to be of minor importance with most of the compounds studied here, since "titration" experiments (unpublished), in which the plasma concentration of one compound is raised from zero in the presence of a constant concentration of the other compound, have shown that diodrast is more effective in depressing the clearance of $\mathrm{p}$-hydroxyhippuric and p-aminohippuric acid than these compounds are in depressing the diodrast clearance. Consequently we have designed our experiments so that diodrast was introduced into the blood after a series of clearance determinations with the hippuric acid derivatives. Thus there are afforded three methods of calculation :

1. Comparison of successive clearances, wherein the clearance $\left(C_{X}\right)$ of that compound $(X)$ with either negligible or least depressive power is measured alone in 3 successive 10-minute periods, followed by a similar 3-period clearance $\left(C_{Y}\right)$ determination of the second compound $(Y)$, simultaneously with $C_{\mathbf{X}}$, an interval of 20 minutes elapsing between the first and second series of clearances.

In such experiments, the plasma concentration of $\mathrm{X}$ has been maintained at the lowest levels compatible with analytical accuracy; for o-, m-, and p-hydroxyhippuric acids this is 2 to $4 \mathrm{mgm}$. per $100 \mathrm{ml}$., for $\mathrm{m}$ - and p-aminohippuric acid and p-acetylaminohippuric acid, 1 to $3 \mathrm{mgm}$. per 100 ml. Hippuran and diodrast have been used in plasma concentrations of 1 to $2 \mathrm{mgm}$. of iodine per $100 \mathrm{ml}$. With these plasma concentrations, 
it is believed that the diodrast clearance is not appreciably depressed by the hippuric acid derivatives.

In this method, precaution must be taken to insure that the renal blood flow remains as constant as possible throughout the two series of observations, and that absolute errors in timing and collection of urine samples are reduced to a minimum. One disturbing physiological factor is excessive hydration to produce diuresis, which tends to cause renal hyperemia in the dog. It has been our experience that the renal plasma flow does not become constant after excessive hydration until the urine flow has, fallen to below $3 \mathrm{ml}$. per minute $(15 \mathrm{kgm} . \mathrm{dog})$ and even then progressive reduction throughout 5 or 6 periods implies regression of hyperemia. At urine flows below 1.0 ml. per minute, accurate timing and complete collection of urine samples are difficult.

2. All the above errors may be avoided in some measure by comparing the successive $\mathrm{C}_{\mathbf{X}} / \mathrm{C}_{\mathrm{Cr}_{1}}$ and $\mathrm{C}_{\mathrm{Y}} / \mathrm{C}_{\mathrm{Cr}_{2}}$ clearance ratios (Column 8 , Table IV and Column 9, Table $\mathrm{V}$ ) in the sense $\mathrm{C}_{\mathrm{X}} / \mathrm{C}_{\mathrm{Cr}_{1}}:: \mathrm{C}_{\mathrm{Y}} / \mathrm{C}_{\mathrm{Cr}_{2}}$ (where $\mathrm{C}_{\mathrm{Cr}_{1}}$ is the creatinine clearance in the first 3 periods and $\mathrm{C}_{\mathrm{Cr}_{2}}$ the creatinine clearance in the second 3 periods). Alternatively, the inulin $\left(\mathrm{C}_{\mathrm{IN}}\right)$ or mannitol $\left(\mathrm{C}_{\mathbf{M}}\right)$ clearance ratio is used for this purpose in man. Here errors in timing and urine collection affect $\mathrm{C}_{\mathbf{X}}$ and $\mathrm{C}_{\mathrm{CX}_{1}}$ (or $\mathrm{C}_{\mathrm{Y}}$ and $\mathrm{C}_{\mathrm{Cr}_{2}}$ ) equally and hence cancel out.

Moreover, in the dog the filtration rate, $\mathrm{C}_{\mathrm{Cr}}$, tends to vary with the renal plasma flow, $C_{X}$ (or $C_{Y}$ where $C_{Y}$ is approximately equal to $C_{X}$ ), the filtration fraction $\mathrm{C}_{\mathrm{Cr}_{r}} / \mathrm{C}_{\mathbf{X}}$ changing only slightly with marked changes in $C_{x}$. Consequently, changes in the renal plasma flow tend to be eliminated when one compares the successive clearance ratios. Indeed, for the dog, we consider this to be a much more accurate method of comparison than method 1.

In man, the filtration fraction varies inversely as the renal plasma flow and the only advantage of this method, as compared with the preceding one, is the elimination of timing and collection errors.

3. Where it is known that $X$ does not depress the clearance of $Y$, an adequate concentration of $\mathrm{X}$ may be maintained in the plasma throughout the determination of the $C_{Y}$ so that there is avail- able the simultaneous clearance ratio, $C_{\mathbf{X}} / C_{\mathbf{Y}}$, in the second series of observations. So far as errors, other than possible mutual depression of clearances are concerned, this is of course the most accurate method of the three.

Where available, all three ratios are reported in Tables IV and V.

Blanks in plasma and cells. Because of the small order of magnitude of the blanks involved in both plasma and cells, these values were determined additively: i.e., a known quantity of substance under determination was added to a suitable filtrate of plasma or cells (see Methods) and the blank determined by difference. Since it is possible that substances present in plasma filtrates may inhibit color development, in some instances at least 3 concentrations were added to aliquots of the same plasma filtrate and the resulting apparent concentrations plotted against the theoretic values. If no inhibition of color development is present, the 3 determinations should fall along a straight line with a slope of 1.0 and which either extrapolates through the zero coordinates or to a positive intercept equal to the value of a constant blank. (Such inhibition has been observed only on m-hydroxy hippuric acid as described under Methods.)

Determination of the blank was, with few exceptions, carried out in each clearance experiment on any animal or man, and an appropriate correction applied to the analytical data. A tabulation of these blank values is given in Table $\mathrm{I}$. The blank values for the p-amino coupling reaction are so small that they can be neglected with plasma concentrations of $1.5 \mathrm{mgm}$. per cent or higher of p-aminohippuric acid, etc.

Blank excretion. Twelve examinations of timed urine samples from 7 individuals indicate an excretion rate of chromogenic material behaving like p-aminohippuric acid, of 0.002 to $0.036 \mathrm{mgm}$. per minute (average 0.0083). Since at a plasma concentration of $2 \mathrm{mgm}$. per cent and with a clearance of $600 \mathrm{ml}$. per minute, UV $=12 \mathrm{mgm}$. per minute, this blank can be wholly neglected in clearance determinations. The identity of the compound or compounds giving the color reaction is unknown. On acid hydrolysis, the UV blank increases slightly, but remains at negligible values. The blank excretion for both "apparent" free p-aminohippuric acid and acid-hydrolyzable, 
TABLE I

Blanks in plasma and cells in dog and man

\begin{tabular}{|c|c|c|c|c|c|c|c|c|}
\hline \multirow{3}{*}{ Compound } & \multicolumn{4}{|c|}{ Dog } & \multicolumn{4}{|c|}{ Man } \\
\hline & \multicolumn{2}{|c|}{ Plasma } & \multicolumn{2}{|c|}{ Cells } & \multicolumn{2}{|c|}{ Plasma } & \multicolumn{2}{|c|}{ Cells } \\
\hline & $\begin{array}{l}\text { No. of } \\
\text { determi- } \\
\text { nations }\end{array}$ & Range & $\begin{array}{c}\text { No. of } \\
\text { determi- } \\
\text { nations }\end{array}$ & Range & $\begin{array}{l}\text { No. of } \\
\text { determi- } \\
\text { nations }\end{array}$ & Range & $\begin{array}{c}\text { No. of } \\
\text { determi- } \\
\text { nations }\end{array}$ & Range \\
\hline $\begin{array}{l}\text { o-Hydroxyhippuric acid } \\
\text { o-Hydroxyhippuric acid } \\
\text { m-Hydroxyhippuric acid } \\
\text { p-Hydroxyhippuric acid } \\
\text { m-Aminohippuric acid } \\
\text { p-Aminohippuric acid } \\
\text { p-Aminohippuric acid } \\
\text { p-Acetylaminohippuric } \\
\text { acid } \\
\text { p-Acetylaminohippuric } \\
\text { acid } \\
\text { p-Aminomandelic acid } \\
\text { p-Aminophenylsuccinic } \\
\text { acid } \\
\text { p-Aminophenaceturic } \\
\text { acid } \\
\text { Diodrast } \\
\text { p-Aminobenzoic acid }\end{array}$ & $\begin{array}{r}21 \\
1^{2} \\
5^{2} \\
64 \\
5^{2} \\
11^{8} \\
5^{2} \\
28 \\
2^{8} \\
4^{2} \\
1^{2} \\
32 \\
9^{2} \\
2^{2}\end{array}$ & $\begin{array}{c}\text { mgm. per } \\
100 \text { ml. } \\
0 \text { to } 0.28 \\
0 \\
0.04 \text { to } 0.06 \\
0.1 \text { to } 0.3 \\
0 \text { to } 0.06 \\
0 \\
0 \\
0.08 \text { to } 0.11 \\
\\
0 \text { to } 0.08 \\
0.02 \\
0 \text { to } 0.01 \\
0 \\
0\end{array}$ & $\begin{array}{l}1^{7} \\
1^{3} \\
5^{2,10} \\
2^{3} \\
6^{8}\end{array}$ & $\begin{array}{c}\text { mgm. per } \\
100 \text { ml. } \\
0.38 \\
0.04 \\
0.3 \text { to } 1.97 \\
0 \text { to } 0.02 \\
0 \text { to } 0.18\end{array}$ & $\begin{array}{l}8^{4} \\
4^{2} \\
9^{5} \\
5^{2} \\
3^{5} \\
2^{2} \\
4^{2}\end{array}$ & $\begin{array}{c}m g m . \text { per } \\
100 \text { ml. } \\
0 \text { to } 0.44 \\
0.13 \text { to } 0.26 \\
0 \\
0 \text { to } 0.10 \\
0 \\
0 \\
0 \\
0 \text { to } 0.05\end{array}$ & $\begin{array}{l}6^{8} \\
2^{8} \\
8^{8} \\
2^{8} \\
1^{8} \\
1^{8} \\
1^{8}\end{array}$ & $\begin{array}{c}\text { mgm. per } \\
100 \mathrm{ml} \text {. } \\
0.38 \\
0 \text { to } 0.31 \\
0 \text { to } 0.01 \\
0 \text { to } 0.4 \\
0 \\
0 \\
0 \\
0.17\end{array}$ \\
\hline
\end{tabular}

1 Precipitated with tungstate 1-5.

2 Precipitated with cadmium sulfate 1-15.

3 Diluted 1-2.5 with distilled water; precipitated with cadmium sulfate 1-5.

4 Precipitated with cadmium sulfate 1-5.

- Precipitated with trichloroacetic acid 1-10.

- Diluted 1-2.5 with distilled water; precipitated with trichloroacetic acid 1-10.

7 Diluted 1-2.5 with distilled water; precipitated with tungstate 1-5.

8 Precipitated with trichloroacetic acid 1-5.

- Analyzed by fusion method.

${ }_{10}$ Direct rather than additive determination.

11 This figure applies to man only and confirms Alpert (20). On dogs we have obtained so nearly 100 per cent recovery that no correction has been made for clearance comparison.

12 White (4) gives 0.32 .

conjugated material is similarly low and negligible in the dog. The blank excretion of p-phenols tends to be somewhat larger, but nevertheless can usually be neglected in both species.

Recoveries were determined in plasma by substituting $2 \mathrm{ml}$. of standard solutions of the compound under investigation for $2 \mathrm{ml}$. of water in the process of precipitating $2 \mathrm{ml}$. of plasma. A similar method was used with cells, the concentrated cells being hemolyzed before addition of the standard solution. The appropriate blank value determined on the sample of plasma or cells was deducted in calculating the recovery.

As might be expected, recovery from hemolyzed cells is less than 100 per cent, but it is nevertheless fairly uniform for any one compound. No consistent difference has been found in the behaviour of dog and human plasma and red cells and the respective figures have been averaged in Table II.

In calculating plasma clearances, recovery has been taken at 100 per cent for all compounds except p-hydroxyhippuric acid and diodrast, where the figures 89 and 94 per cent, respectively, were used in man (for dog, see Note 11, Table II). The recovery figures shown in Table II were used in calculating the in vivo distribution between red cells and plasma shown in Table III.

From experience, we have found that it is important, especially when working with relatively unfamiliar compounds, to include a "blank" and a "recovery determination" in each set of experimental analyses.

In vivo distribution between red cells and plasma (Table III). It is known that although diodrast penetrates the red cell to a negligible 
TABLE II

Precipitation recoveries from dog and human plasma and cells

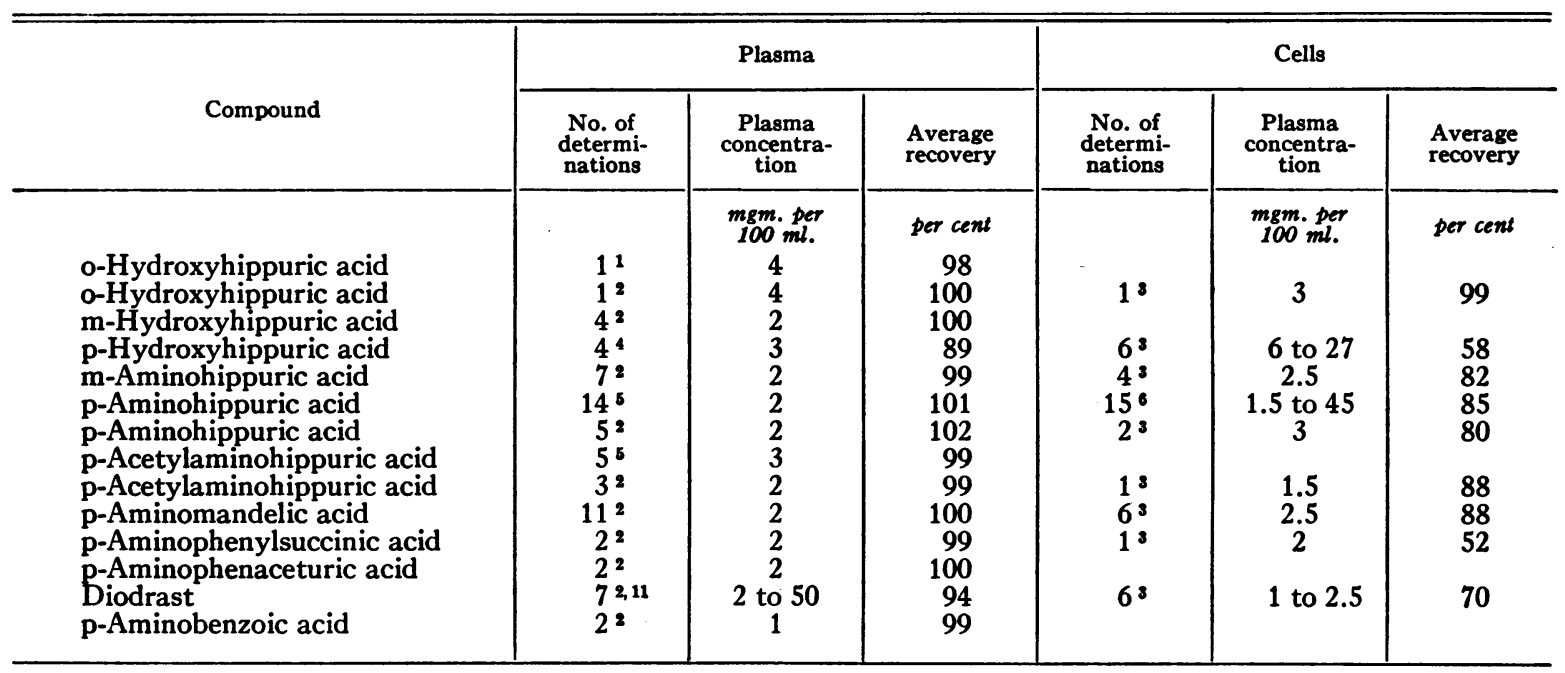

(For footnotes see Table I.)

extent in in vitro mixtures with whole blood (2), considerable penetration occurs in vivo, as demonstrated by White (4), who has concluded that a significant part of the diodrast excreted in the urine in the $\operatorname{dog}$ is extracted from the red cell during the passage of the blood through the kidney. This circumstance would of course tend to give a diodrast clearance above the true value of the renal plasma flow.

We have likewise found that although p-aminohippuric acid does not penetrate the red cell to a significant extent in in vitro mixtures of dog or human blood, there is some penetration in dog blood in vivo. This is true to a greater or less extent of all the compounds listed in Table III, though strangely the ultimate distribution, under conditions where the plasma concentration has been fairly constant for 20 to 90 minutes, is never one of equality per unit of water. This failure to reach an equal distribution per unit of water was also noted by White in the case of diodrast.

TABLE III

In vivo distribution between red cells and plasma

\begin{tabular}{|c|c|c|c|c|c|c|c|c|}
\hline \multirow[b]{2}{*}{ Compound } & \multicolumn{4}{|c|}{ Dog } & \multicolumn{4}{|c|}{ Man } \\
\hline & Method & $\begin{array}{l}\text { No. of } \\
\text { determi- } \\
\text { nations }\end{array}$ & $\begin{array}{l}\text { Plasma } \\
\text { concen- } \\
\text { tration }\end{array}$ & $\frac{\text { Cell }}{\begin{array}{c}\text { Plasma } \\
\text { distri- } \\
\text { bution. }\end{array}}$ & Method & $\begin{array}{c}\text { No. of } \\
\text { determi- } \\
\text { nations }\end{array}$ & $\begin{array}{l}\text { Plasma } \\
\text { concen- } \\
\text { tration }\end{array}$ & $\frac{\text { Cell }}{\begin{array}{c}\text { Plasma } \\
\text { distri- } \\
\text { bution }\end{array}}$ \\
\hline $\begin{array}{l}\text { o-Hydroxyhippuric acid } \\
\text { p-Hydroxyhippuric acid } \\
\text { m-Aminohippuric acid } \\
\text { p-Aminohippuric acid } \\
\text { p-Acetylaminohippuric acid } \\
\text { p-Acetylaminohippuric acid } \\
\text { p-Aminomandelic acid } \\
\text { p-Aminophenylsuccinic acid } \\
\text { Diodrast } \\
\text { Hippuran } \\
\text { Iopax }\end{array}$ & $\begin{array}{l}\text { Direct }^{8} \\
\text { Additive }^{3} \\
\text { Direct }^{8,6}\end{array}$ & $\begin{array}{r}5 \\
1 \\
8 \\
12 \\
6\end{array}$ & $\begin{array}{l}\text { mgm. per } \\
100 \text { ml. } \\
3 \text { to } 10 \\
0.9 \text { to } 2.7 \\
1.5 \text { to } 15\end{array}$ & $\begin{array}{l}\text { per } 100 \\
\text { grams } \\
\text { water } \\
\\
0.3 \\
0.41 \\
0.5\end{array}$ & $\begin{array}{l}\text { Direct }^{7} \\
\text { Additive }^{8} \\
\text { Additive }^{8} \\
\text { Additive }^{2,6} \\
\text { Additive }^{8} \\
\text { Additive }^{3} \\
\text { Additive }^{3} \\
\text { Additive }^{3} \\
\text { Direct }^{\circ}\end{array}$ & $\begin{array}{r}2 \\
8 \\
16 \\
16 \\
4 \\
5 \\
5 \\
10 \\
6\end{array}$ & $\begin{array}{c}\text { mgm. per } \\
100 \text { ml. } \\
1.5 \\
2.5 \text { to } 5.2 \\
2.5 \\
1.5 \\
3 \\
1.5 \\
1.5 \\
1.5 \\
1.5 \text { to } 3.5\end{array}$ & $\begin{array}{c}\text { per } 100 \\
\text { grams } \\
\text { water } \\
0.3 \\
0.16 \\
0 \\
0 \\
0 \\
0 \\
0.16 \\
0.3812 \\
0.49\end{array}$ \\
\hline
\end{tabular}

(For footnotes see Table I.) 
The tendency of all compounds to penetrate the red cell is much less in man than in the dog, and indeed no definite penetration was ever demonstrable in man in the case of m-aminohippuric acid, p-aminohippuric acid, or p-acetylaminohippuric acid. The data on p-aminohippuric acid in Table V include 2 prolonged experiments (11 and 12 periods, respectively) with pyrogenic renal hyperemia (5), a fairly constant concentration of the acid being present in the blood for 3 to 4 hours. Despite this prolonged exposure, no detectable quantity of p-aminohippuric acid was present in the cells. These experiments demonstate that the increase in diodrast and p-aminohippuric acid clearances during pyrogenic hyperemia is referable solely to renal hyperemia and not in any measure to increased extraction of the compound from the red cell. Indeed, the general identity of these clearances indicates that no significant quantity of diodrast moves out of the red cell during the renal passage of the blood.

Comparison of clearances in the dog. The pertinent data on the renal clearances of the substances studied here are given in Table IV.

Essentially identical clearances are shown by m-hydroxyhippuric acid, p-hydroxyhippuric acid, m-aminohippuric acid, $\mathrm{p}$-aminohippuric acid, $\mathrm{p}$ acetylaminohippuric acid, 2-pridone-1-acetic acid, cinnamoylglycine, hippuran, and diodrast.

This conclusion would be reached whether one considered the ratios of successive clearances (Column 7), the ratio of the successive filtration fractions (Column 8), or the ratio of the simultaneous clearances (Column 9), except in the case of cinnamoylglycine the clearance of which appears to be substantially depressed by the presence in the plasma of p-aminohippuric acid.

It is thus clear that the presence of iodine in hippuran (o-iodohippuric acid) is not essential to the process of tubular excretion, but that the necessary properties are possessed by the parent hippuric acid molecule. Similarly, 2-pyridone-1acetic acid (of which a sufficient quantity for only 2 experiments was available) appears to be excreted as efficiently as is the iodo derivative, iopax (5-iodo-2-pyridone-1-acetic acid) (6). The identity of the above clearances conforms with the premise $(7,2)$ that the limiting circumstance in renal excretion of diodrast (or of any one of the above substances) at low plasma concentrations is the effective blood flow to the renal tubules (which supposedly closely approximates the total renal blood flow ${ }^{4}$ ) rather than a limitation in the tubular excretory mechanism.

Clearances significantly lower in the dog than those of diodrast or the above hippuric acid derivatives are shown by o-hydroxyhippuric acid, $\mathrm{p}$-aminophenaceturic acid, iopax, and p-aminobenzoic acid. No interpretation suggests itself to account for the difference in behavior between $\mathrm{o}$-hydroxyhippuric acid and the $\mathrm{p}$ - and $\mathrm{m}$ - derivatives or for the behavior of iopax, though presumably it represents some limitation in the tubular excretory mechanism. p-Aminobenzoic acid, when infused at a constant rate, shows a tendency for the clearance to rise with time, and in 1 experiment where a single injection of $\mathrm{p}$-aminobenzoic acid was given intravenously and the clearance was determined in successive periods on a falling plasma concentration, the clearance rose substantially above that of creatinine. We interpret the rising clearance as reflecting the formation in the body of $\mathrm{p}$-aminohippuric acid from $\mathrm{p}$-aminobenzoic acid. Similarly, p-aminophenylsuccinic acid shows a rising clearance starting in excess of the creatinine clearance, implying metabolism to an unidentified compound having a high clearance value.

p-Aminomandelic acid has a clearance very close to that of creatinine. Whether the discrepancy between the two clearances is attributable to systematic error in the determination of one or the other substances or indicates tubular excretion of the acid cannot be said from the available data. Unfortunately, necessary cessation of work prevented further examination.

Comparison of clearances in man (Table V) ${ }^{5}$. The same methods of comparison as are described

4 White (4) reports the average diodrast renal extraction ratio in the dog as $\mathbf{0 . 7 4}$, while Corcoran, Smith and Page (23) obtained 0.87. More recently Bradley and his coworkers (personal communication) and Stead and his coworkers (personal communication) have independently observed the renal extraction ratio of diodrast in man to averge 0.87 . Phillips et al. (24) report the extraction ratio of $\mathrm{p}$-aminohippuric acid in the dog to be 0.85 .

${ }^{5}$ We aŕe indebted to Dr. William Goldring, Dr. Herbert Chasis, Dr. Hilmert Ranges, and Dr. Stanley Bradley for assistance in the observations on man reported in Table V, and to Miss Martha Barrett and Miss Claire Lawler for the inulin and mannitol determinations. 
TABLE IV

Comparison of successive and simultaneous clearance ratios in the dog

Each experiment consists of 2 series of 3 clearance periods each; in the first series, the clearance of substance X was observed alone, relative to the creatinine clearance, after which substance $Y$ was introduced (second series). Thus there are available the ratio of the successive clearances of $X$ and $Y$ (column 7 ), the ratio of the successive filtration fractions (column 8), and the ratio of the simultaneous clearances (column 9). All clearances have been corrected to 1 square meter body surface area.

\begin{tabular}{c|c|c|c|c|c|c|c|c}
\hline \hline 1 & 2 & 3 & 4 & 5 & 6 & 7 & 8 \\
$\begin{array}{c}\text { Dog } \\
\text { no. }\end{array}$ & Date & $\mathrm{C}_{\mathrm{X}}$ & $\mathrm{C}_{\mathrm{Y}}$ & $\frac{\mathrm{C}_{\mathrm{CR}_{1}}}{\mathrm{C}_{\mathrm{X}}}$ & $\frac{\mathrm{C}_{\mathrm{CR}_{2}}}{\mathrm{C}_{\mathrm{Y}}}$ & $\begin{array}{c}\text { Successive } \\
\text { clearance } \\
\text { ratio } \\
\mathrm{C}_{\mathrm{X}} / \mathrm{C}_{\mathrm{Y}}\end{array}$ & $\begin{array}{c}\mathrm{C}_{\mathrm{CR}_{\mathbf{2}} / \mathrm{C}_{\mathrm{Y}}} \\
\mathrm{C}_{\mathrm{CR}_{\mathbf{I}} / \mathrm{C}_{\mathrm{X}}}\end{array}$ & $\begin{array}{c}\text { Simultaneous } \\
\text { clearance } \\
\text { ratio } \\
\mathrm{C}_{\mathrm{X}} / \mathrm{C}_{\mathrm{Y}}\end{array}$ \\
\hline
\end{tabular}

o-Hydroxyhippuric acid (X) and diodrast (Y)

\begin{tabular}{|c|c|c|c|c|c|c|c|c|}
\hline $\begin{array}{l}3 \\
1 \\
3\end{array}$ & $\begin{array}{l}12-2-40 \\
12-4-40 \\
12-17-41\end{array}$ & $\begin{array}{r}173 \\
84 \\
110\end{array}$ & $\begin{array}{l}208 \\
133 \\
175\end{array}$ & $\begin{array}{l}0.37 \\
0.48 \\
0.52\end{array}$ & $\begin{array}{c}0.26 \\
0.31 \\
0.31\end{array}$ & $\begin{array}{l}0.83 \\
0.63 \\
0.71 \\
0.72\end{array}$ & $\begin{array}{l}0.72 \\
0.64 \\
0.64 \\
0.66\end{array}$ & $\begin{array}{l}0.65 \\
0.41 \\
0.56 \\
0.53\end{array}$ \\
\hline
\end{tabular}

m-Hydroxyhippuric acid $(\mathrm{X})$ and diodrast $(\mathrm{Y})$

\begin{tabular}{|c|c|c|c|c|c|c|c|c|}
\hline $\begin{array}{l}4 \\
1 \\
3 \\
4\end{array}$ & $\begin{array}{l}11-26-41 \\
11-28-41 \\
12-1-41 \\
12-15-41\end{array}$ & $\begin{array}{l}154 \\
150 \\
180 \\
163\end{array}$ & $\begin{array}{l}150 \\
129 \\
170 \\
152\end{array}$ & $\begin{array}{l}0.26 \\
0.20 \\
0.29 \\
0.29\end{array}$ & $\begin{array}{l}0.28 \\
0.24 \\
0.33 \\
0.30 \\
\text { A }\end{array}$ & $\begin{array}{l}0.92 \\
1.10 \\
1.11 \\
1.10 \\
\text { e } 1.03\end{array}$ & $\begin{array}{l}1.05 \\
1.22 \\
1.10 \\
1.04 \\
1.10\end{array}$ & $\begin{array}{l}0.97 \\
1.16 \\
1.14 \\
1.06 \\
1.08\end{array}$ \\
\hline \multicolumn{9}{|c|}{ p-Hydroxyhippuric acid $(\mathrm{X})$ and diodrast $(\mathrm{Y})$} \\
\hline $\begin{array}{l}4 \\
3 \\
3 \\
5\end{array}$ & $\begin{array}{r}10-7-40 \\
10-16-40 \\
11-13-40 \\
5-24-40\end{array}$ & $\begin{array}{l}196 \\
246 \\
235 \\
150\end{array}$ & $\begin{array}{l}202 \\
258 \\
230 \\
152\end{array}$ & $\begin{array}{l}0.25 \\
0.30 \\
0.29 \\
0.37\end{array}$ & $\begin{array}{l}0.25 \\
0.29 \\
0.28 \\
0.37 \\
\quad \text { A }\end{array}$ & $\begin{array}{l}0.92 \\
0.95 \\
1.02 \\
\\
\end{array}$ & $\begin{array}{l}0.98 \\
0.98 \\
0.98 \\
\\
0.98\end{array}$ & $\begin{array}{l}0.99 \\
0.99\end{array}$ \\
\hline \multicolumn{9}{|c|}{ p-Hydroxyhippuric acid (X) and hippuran (Y) } \\
\hline $\begin{array}{l}2 \\
3 \\
4\end{array}$ & $\begin{array}{r}12-9-40 \\
1-7-41 \\
4-24-41\end{array}$ & $\begin{array}{l}170 \\
170 \\
159\end{array}$ & $\begin{array}{l}160 \\
178 \\
128\end{array}$ & $\begin{array}{l}0.29 \\
0.31 \\
0.31\end{array}$ & $\begin{array}{l}0.28 \\
0.31 \\
0.37 \\
\quad \text { A }\end{array}$ & $\begin{array}{l}1.08 \\
0.99 \\
(1.24)^{2} \\
\mathrm{e} \\
1.03\end{array}$ & $\begin{array}{l}0.98 \\
1.01 \\
(1.18)^{2} \\
1.00\end{array}$ & $\begin{array}{l}0.92 \\
0.83 \\
1.01 \\
0.92\end{array}$ \\
\hline \multicolumn{9}{|c|}{ p-Hydroxyhippuric acid (X) and p-acetylaminohippuric acid (Y) } \\
\hline 4 & $12-11-40$ & 174 & 139 & 0.31 & 0.35 & $(1.25)^{1}$ & $(1.12)^{1}$ & 0.96 \\
\hline \multicolumn{9}{|c|}{ m-Aminohippuric acid $(\mathrm{X})$ and diodrast $(\mathrm{Y})$} \\
\hline $\begin{array}{l}3 \\
2 \\
3 \\
2\end{array}$ & $\begin{array}{l}10-24-41 \\
10-27-41 \\
11-3-41 \\
12-3-41\end{array}$ & $\begin{array}{l}213 \\
149 \\
146 \\
143\end{array}$ & $\begin{array}{r}271 \\
143 \\
173 \\
. \quad 148\end{array}$ & $\begin{array}{l}0.25 \\
0.26 \\
0.28 \\
0.29\end{array}$ & $\begin{array}{l}0.23 \\
0.25 \\
0.28 \\
0.31 \\
\quad \text { A }\end{array}$ & $\begin{array}{l}(0.79)^{1} \\
1.05 \\
(0.84)^{1} \\
0.90 \\
\text { e } 0.98\end{array}$ & $\begin{array}{l}(0.90)^{1} \\
0.94 \\
1.00 \\
1.07 \\
1.00\end{array}$ & $\begin{array}{l}1.01 \\
0.97 \\
1.00 \\
1.07 \\
1.01\end{array}$ \\
\hline \multicolumn{9}{|c|}{ p-Aminohippuric acid $(X)$ and diodrast $(Y)$} \\
\hline $\begin{array}{l}1 \\
2 \\
3 \\
4 \\
4 \\
2\end{array}$ & $\begin{array}{r}1-9-41 \\
1-13-41 \\
1-16-41 \\
5-12-41 \\
6-12-41 \\
10-1-41\end{array}$ & $\begin{array}{l}135 \\
169 \\
197 \\
154 \\
140 \\
179\end{array}$ & $\begin{array}{l}146 \\
174 \\
207 \\
149 \\
122 \\
162\end{array}$ & $\begin{array}{l}0.28 \\
0.28 \\
0.33 \\
0.32 \\
0.30 \\
0.26\end{array}$ & $\begin{array}{l}0.26 \\
0.25 \\
0.32 \\
0.31 \\
0.33 \\
0.27 \\
\text { A }\end{array}$ & $\begin{array}{l}0.93 \\
0.97 \\
0.95 \\
1.03 \\
1.15 \\
1.11 \\
\text { e } 1.02\end{array}$ & $\begin{array}{l}0.93 \\
0.92 \\
0.96 \\
0.96 \\
1.10 \\
1.01 \\
0.98\end{array}$ & $\begin{array}{l}0.83 \\
0.93 \\
0.94 \\
0.99 \\
1.04 \\
1.07 \\
0.97\end{array}$ \\
\hline
\end{tabular}


TABLE IV-Continued

\begin{tabular}{|c|c|c|c|c|c|c|c|c|}
\hline $\begin{array}{c}1 \\
\text { Dog } \\
\text { no. }\end{array}$ & Date & $\begin{array}{c}3 \\
\mathrm{c}_{\mathrm{X}}\end{array}$ & $\begin{array}{c}4 \\
\mathrm{C}_{\mathrm{Y}}\end{array}$ & $\begin{array}{c}5 \\
\frac{\mathrm{C}_{\mathrm{CR}_{1}}}{\mathrm{C}_{\mathrm{X}}}\end{array}$ & $\begin{array}{c}6 \\
\frac{c_{C_{2}}}{c_{Y}}\end{array}$ & $\begin{array}{c}7 \\
\text { Successive } \\
\text { clearance } \\
\text { ratio } \\
\mathrm{C}_{\mathbf{X}} / \mathbf{C}_{\mathbf{Y}}\end{array}$ & $\begin{array}{c}8 \\
\frac{C_{C_{2} / C_{Y}}}{C_{C_{R_{1}} / C_{X}}}\end{array}$ & $\begin{array}{c}9 \\
\substack{\text { Simultaneous } \\
\text { clearance } \\
\text { ratio } \\
\mathrm{C}_{\mathrm{X}} / \mathrm{C}_{\mathrm{Y}}}\end{array}$ \\
\hline \multicolumn{9}{|c|}{ p-Acetylaminohippuric acid $(\mathrm{X})$ and diodrast $(\mathrm{Y})$} \\
\hline $\begin{array}{l}3 \\
1 \\
2 \\
4 \\
3\end{array}$ & $\begin{array}{r}12-16-40 \\
12-19-40 \\
1-2-41 \\
12-22-41 \\
12-23-41\end{array}$ & $\begin{array}{l}193 \\
129 \\
168 \\
163 \\
252\end{array}$ & $\begin{array}{l}256 \\
130 \\
150 \\
155 \\
256\end{array}$ & $\begin{array}{l}0.29 \\
0.27 \\
0.24 \\
0.25 \\
0.29\end{array}$ & $\begin{array}{l}0.23 \\
0.29 \\
0.27 \\
0.26 \\
0.30 \\
\text { A }\end{array}$ & $\begin{array}{c}(0.75)^{1} \\
0.99 \\
1.12 \\
0.97 \\
0.96 \\
\text { ge } 1.01\end{array}$ & $\begin{array}{c}(0.79)^{1} \\
1.10 \\
1.06 \\
1.01 \\
1.01 \\
1.04\end{array}$ & $\begin{array}{l}0.94 \\
0.96 \\
0.94 \\
1.02 \\
0.97\end{array}$ \\
\hline
\end{tabular}

p-Aminophenaceturic acid $(\mathrm{X})$ and diodrast $(\mathrm{Y})$

\begin{tabular}{|c|c|c|c|c|c|c|c|}
\hline $\begin{array}{l}1 \\
4 \\
2 \\
4\end{array}$ & $\begin{array}{r}22-6-42 \\
2-10-42 \\
2-12-42 \\
4-17-42\end{array}$ & $\begin{array}{l}104 \\
145 \\
180 \\
139\end{array}$ & $\begin{array}{l}168 \\
187 \\
187\end{array}$ & $\begin{array}{l}0.31 \\
0.32 \\
0.28 \\
0.39\end{array}$ & 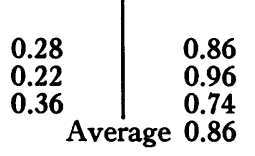 & $\begin{array}{l}0.88 \\
0.79 \\
0.79 \\
0.82\end{array}$ & $\begin{array}{l}0.78 \\
0.68 \\
0.67 \\
0.71\end{array}$ \\
\hline \multicolumn{8}{|c|}{ Iopax (X) and p-hydroxyhippuric acid (Y) } \\
\hline $\begin{array}{l}4 \\
1 \\
4\end{array}$ & $\begin{array}{l}1-20-41 \\
1-22-41 \\
4-28-41\end{array}$ & $\begin{array}{r}124 \\
54 \\
166\end{array}$ & $\begin{array}{l}148 \\
116 \\
261\end{array}$ & $\begin{array}{l}0.41 \\
0.61 \\
0.38\end{array}$ & 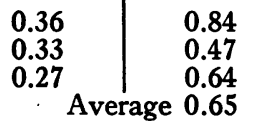 & $\begin{array}{l}0.87 \\
0.54 \\
0.70 \\
0.70\end{array}$ & $\begin{array}{l}0.97 \\
0.69 \\
0.81 \\
0.82\end{array}$ \\
\hline \multicolumn{8}{|c|}{ 2-Pyridone-1-acetic acid (X) and p-aminohippuric acid (Y) } \\
\hline $\begin{array}{l}3 \\
1\end{array}$ & $\begin{array}{l}5-7-42 \\
5-12-42\end{array}$ & $\begin{array}{l}162 \\
173\end{array}$ & $\begin{array}{l}185 \\
143\end{array}$ & $\begin{array}{l}0.34 \\
0.21\end{array}$ & \begin{tabular}{l|l}
0.39 & 0.88 \\
0.25 & 1.21 \\
\multicolumn{2}{l}{ Average 1.04}
\end{tabular} & $\begin{array}{l}1.12 \\
1.17 \\
1.15\end{array}$ & $\begin{array}{l}0.83 \\
1.13 \\
0.98\end{array}$ \\
\hline \multicolumn{8}{|c|}{ Cinnamoylglycine $(\mathrm{X})$ and p-aminohippuric acid $(\mathrm{Y})$} \\
\hline $\begin{array}{l}1 \\
3\end{array}$ & $\begin{array}{l}3-6-42 \\
4-27-42\end{array}$ & $\begin{array}{l}106 \\
153\end{array}$ & $\begin{array}{l}127 \\
139\end{array}$ & $\begin{array}{l}0.27 \\
0.26\end{array}$ & 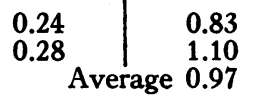 & $\begin{array}{l}0.92 \\
0.90 \\
0.91\end{array}$ & $\begin{array}{l}0.70 \\
0.70 \\
0.70\end{array}$ \\
\hline \multicolumn{8}{|c|}{ p-Aminophenylsuccinic acid $(X)$ and creatinine $(Y)$} \\
\hline $\begin{array}{l}2 \\
4\end{array}$ & $\begin{array}{l}11-5-41 \\
11-7-41\end{array}$ & \multicolumn{5}{|c|}{$\begin{array}{l}\text { Simultaneous values of } C_{X} / C_{Y} \text { rose from } 1.05 \text { to } 1.47 \\
\text { Simultaneous values of } C_{X} / C_{Y} \text { rose from } 1.61 \text { to } 1.86\end{array}$} & \\
\hline \multicolumn{8}{|c|}{ p-Aminobenzoic acid $(\mathrm{X})$ and creatinine $(\mathrm{Y})$} \\
\hline $\begin{array}{l}1 \\
2\end{array}$ & $\begin{array}{l}11-10-41 \\
11-13-41\end{array}$ & 25 & $\begin{array}{c}42 \\
4 \\
\text { ultan }\end{array}$ & $\begin{array}{l}0.59 \\
\text { lues of }\end{array}$ & rose from 1.10 to 1 & & \\
\hline \multicolumn{8}{|c|}{ p-Aminomandelic acid $(X)$ and creatinine $(Y)$} \\
\hline $\begin{array}{l}1 \\
4 \\
1\end{array}$ & $\begin{array}{l}10-8-41 \\
10-16-41 \\
10-22-41\end{array}$ & $\begin{array}{l}38 \\
54 \\
37\end{array}$ & $\begin{array}{l}33 \\
52 \\
36\end{array}$ & $\begin{array}{l}1.15 \\
1.04 \\
1.03 \\
1.07\end{array}$ & & & \\
\hline
\end{tabular}

1 Clearance of $\mathrm{X}$ increased in second series of test, indicating increased renal plasma flow and invalidating comparison of successive clearances or successive clearance ratios.

2 Clearance of $\mathrm{X}$ increased so markedly in second series of test that discrepancy is clearly attributed to receding hyperemia. 
TABLE V

Comparison of successive and simultaneous clearance ratios in man

Each experiment consists of 2 series of clearance periods; in the first series, the clearance of substance $\mathrm{X}$ was observed alone, after which substance $Y$ was introduced (second series). Thus there are available, relative to the inulin (or mannitol) clearance, the ratio of the successive clearances (column 9), the ratio of the successive filtration fractions (column 10), and the ratio of the simultaneous clearances (column 11). All clearances have been corrected to 1.73 square meter surface area.

\begin{tabular}{c|c|c|c|c|c|c|c|c|c|c}
\hline \hline 1 & 2 & 3 & 4 & 5 & 6 & 7 & 8 & 9 & 10 & 11 \\
Subject & Date & $\begin{array}{c}\text { Number } \\
\text { of } \\
\text { determi- } \\
\text { nations }\end{array}$ & $\mathrm{C}_{\mathrm{X}}$ & $\mathrm{C}_{\mathrm{Y}}$ & $\frac{\mathrm{C}_{\mathrm{IN}}}{\mathrm{C}_{\mathrm{X}}}$ & $\frac{\mathrm{C}_{\mathrm{IN} \mathrm{N}_{2}}}{\mathrm{C}_{\mathrm{Y}}}$ & $\begin{array}{c}\text { Succes- } \\
\text { sive } \\
\text { clearance } \\
\text { ratio } \\
\mathrm{C}_{\mathrm{X}} / \mathrm{C}_{\mathrm{Y}}\end{array}$ & $\frac{\mathrm{C}_{\mathrm{IN} \mathrm{N}_{2} / \mathrm{C}_{\mathrm{Y}}}}{\mathrm{C}_{\mathrm{IN} \mathrm{N}_{1}} / \mathrm{C}_{\mathrm{X}}}$ & $\begin{array}{c}\text { Simulta- } \\
\text { neous } \\
\text { clearance } \\
\text { ratio } \\
\mathrm{C}_{\mathrm{X}} / \mathrm{C}_{\mathrm{Y}}\end{array}$ & Diagnosis \\
\hline
\end{tabular}

o-Hydroxyhippuric acid $(\mathrm{X})$ and diodrast $(\mathrm{Y})$

\begin{tabular}{l|r|l|l|l|l|l|l|l|l|l}
\hline I.N. & $12-13-40$ & 8 & 269 & 428 & 0.35 & 0.21 & 0.63 & 0.68 & 0.61 & Subdeltoid bursitis \\
J.K. & $4-4-41$ & 6 & 487 & 686 & 0.23 & 0.16 & 0.70 & 0.69 & & Pneumonia \\
& & & & & & & Average 0.66 & 0.68 & & \\
\hline
\end{tabular}

p-Hydroxyhippuric acid $(\mathrm{X})$ and diodrast $(\mathrm{Y})$

\begin{tabular}{|c|c|c|c|c|c|c|c|c|c|c|}
\hline $\begin{array}{l}\text { S.W. } \\
\text { W.O. } \\
\text { C.A. } \\
\text { M.J. } \\
\text { B.D. } \\
\text { M.J. } \\
\text { V.B. }\end{array}$ & $\begin{array}{r}11-21-40 \\
3-31-41 \\
4-9-41 \\
4-18-41 \\
4-21-41 \\
4-23-41 \\
1-2-41\end{array}$ & $\begin{array}{r}10 \\
6 \\
6 \\
6 \\
6 \\
6 \\
8\end{array}$ & $\begin{array}{l}364 \\
532 \\
702 \\
478 \\
544 \\
487 \\
\text { Pyrog }\end{array}$ & $\begin{array}{l}375 \\
845 \\
692 \\
472 \\
574 \\
432 \\
\text { enic hy }\end{array}$ & $\begin{array}{c}0.20 \\
0.24 \\
0.18 \\
0.23 \\
0.17 \\
0.22 \\
\text { seremia }\end{array}$ & $\begin{array}{l}0.21 \\
0.15 \\
0.19 \\
0.23 \\
0.17 \\
0.24 \\
\\
\text { Avera: }\end{array}$ & $\begin{array}{c}0.97 \\
(0.63)^{1} \\
1.02 \\
1.01 \\
1.13 \\
\\
1.03\end{array}$ & $\begin{array}{l}1.02 \\
(0.63)^{1} \\
1.06 \\
0.99 \\
1.11 \\
1.05\end{array}$ & $\begin{array}{l}0.98 \\
1.00\end{array}$ & $\begin{array}{l}\text { Hypertension } \\
\text { Alcoholism } \\
\text { Syphilis } \\
\text { Hypertension } \\
\text { Multiple sclerosis } \\
\text { Hypertension } \\
\text { Grippe }\end{array}$ \\
\hline
\end{tabular}

p-Hydroxyhippuric acid (X) and hippuran (Y)

\begin{tabular}{l|l|l|l|l|l|l|l|l|l|l}
\hline P.R. & $4-28-41$ & 6 & 554 & 586 & 0.18 & 0.15 & 0.94 & 0.87 & & Gastric ulcer \\
H.B. & $4-30-41$ & 6 & 722 & 670 & 0.18 & 0.17 & 1.08 & 0.99 & & Arthritis \\
A.R. & $5-9-41$ & 6 & 395 & 391 & 0.23 & 0.22 & 1.01 & 0.92 & & Rheumatic fever \\
\hline
\end{tabular}

m-Aminohippuric acid $(\mathrm{X})$ and diodrast $(\mathrm{Y})$

\begin{tabular}{|c|c|c|c|c|c|c|c|c|c|c|}
\hline $\begin{array}{l}\text { K.F.* } \\
\text { L.R.** } \\
\text { A.C.** } \\
\text { L.A.* }\end{array}$ & $\begin{array}{l}11-7-41 \\
11-10-41 \\
12-5-41 \\
11-14-41\end{array}$ & $\begin{array}{l}6 \\
6 \\
4 \\
6\end{array}$ & $\begin{array}{l}686 \\
474 \\
406 \\
592\end{array}$ & $\begin{array}{l}462 \\
481 \\
528\end{array}$ & $\begin{array}{l}0.17 \\
0.19 \\
0.17 \\
0.18\end{array}$ & $\begin{array}{l}0.21 \\
0.14 \\
0.18 \\
\text { Averag }\end{array}$ & $\begin{array}{l}1.03 \\
0.84 \\
1.12 \\
0.99\end{array}$ & $\begin{array}{l}1.11 \\
0.81 \\
1.01 \\
0.97\end{array}$ & $\begin{array}{l}0.99 \\
0.96 \\
1.02 \\
0.99\end{array}$ & $\begin{array}{l}\text { Essential hypertension } \\
\text { Cholelithiasis } \\
\text { Nephritis } \\
\text { Bronchitis }\end{array}$ \\
\hline \multicolumn{11}{|c|}{ p-Aminohippuric acid $(\mathrm{X})$ and diodrast $(\mathrm{Y})$} \\
\hline $\begin{array}{l}\text { H.B. } \\
\text { J.L.: } \\
\text { J.M. } \\
\text { R.C. } \\
\text { J.B. } \\
\text { C.B. } \\
\text { P.H. } \\
\text { C.B. } \\
\text { S.L.** } \\
\text { F.T.* } \\
\text { C.BI.** } \\
\text { P.L.* } \\
\text { M.A.* }\end{array}$ & $\begin{array}{c}4-2-41 \\
4-14-41 \\
4-16-41 \\
5-13-41 \\
5-14-41 \\
5-16-41 \\
10-13-41 \dagger \\
10-20-41 \dagger \\
7-16-42 \\
1-6-43 \dagger \\
1-22-43 \dagger \\
1-27-43 \dagger \\
2-12-43\end{array}$ & $\begin{array}{r}5 \\
6 \\
6 \\
6 \\
6 \\
6 \\
12 \\
11 \\
6 \\
7 \\
7 \\
7 \\
2\end{array}$ & $\begin{array}{c}695 \\
509 \\
442 \\
598 \\
588 \\
491 \\
\text { Hype } \\
\text { Hype } \\
574 \\
188 \\
334 \\
424 \\
30\end{array}$ & $\begin{array}{r}649 \\
591 \\
534 \\
609 \\
603 \\
511 \\
\text { emia } \\
\text { emia } \\
604 \\
138 \\
309 \\
452 \\
26\end{array}$ & $\begin{array}{l}0.23 \\
0.24 \\
0.22 \\
0.14 \\
0.20 \\
0.23 \\
\\
\\
0.18 \\
0.23 \\
0.21 \\
0.20 \\
0.67\end{array}$ & $\begin{array}{l}0.18 \\
0.20 \\
0.20 \\
0.14 \\
0.18 \\
0.21 \\
\\
0.18 \\
0.31 \\
0.22 \\
0.19 \\
0.70\end{array}$ & $\begin{array}{l}1.07 \\
0.86 \\
0.83 \\
0.98 \\
0.98 \\
0.96\end{array}$ & $\begin{array}{l}0.77 \\
0.81 \\
0.92 \\
0.60 \\
0.89 \\
0.91\end{array}$ & $\begin{array}{l}0.98 \\
1.01 \\
1.02 \\
0.99 \\
1.32 \\
1.08 \\
0.94 \\
1.06\end{array}$ & $\begin{array}{l}\text { Arthritis } \\
\text { Grippe } \\
\text { Lumbar sacral sprain } \\
\text { G.I. pathology } \\
\text { Glomerular nephritis } \\
\text { Essential hypertension } \\
\text { Chronic duodenitis } \\
\text { Essential hypertension } \\
\text { Duodenal ulcer } \\
\text { Glomerular nephritis } \\
\text { Essential hypertension } \\
\text { Essential hypertension } \\
\text { Chronic difuse glomerular }\end{array}$ \\
\hline J.V.* & $2-15-43$ & 6 & 678 & 512 & 0.19 & 0.22 & 1.32 & 1.15 & 1.10 & $\begin{array}{l}\text { nephritis } \\
\text { Chronic diffuse glomerular } \\
\text { nephritis }\end{array}$ \\
\hline H.L.* & $1-15-43 \dagger$ & 7 & 39 & 51 & 0.28 & 0.22 & & & 0.78 & Terminal chronic diffuse \\
\hline F.T.* & $1-18-43 \dagger$ & 6 & 178 & 158 & 0.19 & 0.22 & & & 1.12 & $\begin{array}{l}\text { Chronic diffuse glomerular } \\
\text { nephritis }\end{array}$ \\
\hline
\end{tabular}


TABLE v-Continued

\begin{tabular}{c|c|c|c|c|c|c|c|c|c|c}
\hline 1 & 2 & 3 & 4 & 5 & 6 & 7 & 8 & 9 & 10 & 11 \\
Subject & Date & $\begin{array}{c}\text { Number } \\
\text { def } \\
\text { nations }\end{array}$ & $\mathrm{C}_{\mathrm{X}}$ & $\mathrm{C}_{\mathrm{Y}}$ & $\frac{\mathrm{C}_{\mathrm{IN}}}{\mathrm{C}_{\mathrm{X}}}$ & $\frac{\mathrm{C}_{\mathrm{IN}}}{\mathrm{C}_{\mathrm{Y}}}$ & $\begin{array}{c}\text { Succes- } \\
\text { sive } \\
\text { clearance } \\
\text { ratio } \\
\mathrm{C}_{\mathrm{X}} / \mathrm{C}_{\mathrm{Y}}\end{array}$ & $\frac{\mathrm{C}_{\mathrm{IN}} / \mathrm{C}_{\mathrm{Y}}}{\mathrm{C}_{\mathrm{IN}} / \mathrm{C}_{\mathrm{X}}}$ & $\begin{array}{c}\text { Simulta- } \\
\text { neous } \\
\text { clearance } \\
\text { ratio } \\
\mathrm{C}_{\mathrm{X}} / \mathrm{C}_{\mathrm{Y}}\end{array}$ & Diagnosis \\
\hline
\end{tabular}

p-Aminohippuric acid (X) and diodrast $(\mathrm{Y})$-Continued

\begin{tabular}{|c|c|c|c|c|c|c|c|c|c|c|}
\hline $\begin{array}{l}\text { A.P.* } \\
\text { A.P. } \\
\text { C.S.* }\end{array}$ & $\begin{array}{l}2-19-43 \\
2-22-43 \\
2-24-43\end{array}$ & $\begin{array}{l}4 \\
6 \\
6\end{array}$ & $\begin{array}{r}415 \\
473 \\
11\end{array}$ & $\begin{array}{r}437 \\
536 \\
10\end{array}$ & $\begin{array}{l}0.20 \\
0.19 \\
0.40\end{array}$ & $\begin{array}{l}0.20 \\
0.17 \\
0.82\end{array}$ & $\begin{array}{l}0.95 \\
0.88 \\
1.07\end{array}$ & $\begin{array}{c}1.00 \\
0.91 \\
(2.04)^{1}\end{array}$ & $\begin{array}{l}1.02 \\
0.94 \\
1.06\end{array}$ & $\begin{array}{l}\text { Essential hypertension } \\
\text { Essential hypertension } \\
\text { Chronic diffuse glomerular }\end{array}$ \\
\hline $\begin{array}{l}\text { J.O.* } \\
\text { J.O.* } \\
\text { M.S.** } \\
\text { M.S.* }\end{array}$ & $\begin{array}{l}2-26-43 \\
3-1-43 \\
3-5-43 \\
3-8-43\end{array}$ & $\begin{array}{l}6 \\
6 \\
6 \\
6\end{array}$ & $\begin{array}{l}410 \\
393 \\
504 \\
447\end{array}$ & $\begin{array}{l}390 \\
407 \\
484 \\
340\end{array}$ & $\begin{array}{l}0.22 \\
0.23 \\
0.18 \\
0.19\end{array}$ & $\begin{array}{l}0.24 \\
0.20 \\
0.21 \\
0.24\end{array}$ & $\begin{array}{l}1.05 \\
0.97 \\
1.04 \\
1.32\end{array}$ & $\begin{array}{l}1.08 \\
0.89 \\
1.14 \\
1.30\end{array}$ & $\begin{array}{l}1.00 \\
0.88 \\
0.97 \\
1.21\end{array}$ & $\begin{array}{l}\text { Cardiorenal disease } \\
\text { Cardiorenal disease } \\
\text { Cardiac hypertension } \\
\text { Acute diffuse glomerular }\end{array}$ \\
\hline $\begin{array}{l}\text { A.S.* } \\
\text { A.S.* } \\
\text { A.S.* } \\
\text { P.M.c.* } \\
\text { P.C.* } \\
\text { P.C.* } \\
\text { B.V.* } \\
\text { M.R. } \\
\text { M.R.* } \\
\text { W.D. } \\
\text { A.W.* }\end{array}$ & $\begin{array}{l}5-5-43 \\
5-10-43 \\
5-14-43 \\
5-17-43 \dagger \\
5-19-43 \dagger \\
5-21-43 \dagger \\
5-24-43 \dagger \\
6-2-43 * \\
6-4-43 \dagger \\
6-7-43 \dagger \\
6-9-43 \dagger\end{array}$ & $\begin{array}{r}9 \\
12 \\
6 \\
6 \\
10 \\
3 \\
3 \\
3 \\
3 \\
3 \\
3\end{array}$ & $\begin{array}{l}850 \\
954 \\
920 \\
370 \\
442 \\
446 \\
787 \\
784 \\
762 \\
453 \\
368\end{array}$ & $\begin{array}{l}922 \\
847 \\
962 \\
391 \\
470 \\
422 \\
684 \\
605 \\
675 \\
422 \\
370\end{array}$ & $\begin{array}{l}0.13 \\
0.12 \\
0.11 \\
0.19 \\
0.16 \\
0.18 \\
0.14 \\
0.13 \\
0.13 \\
0.18 \\
0.19\end{array}$ & $\begin{array}{l}0.12 \\
0.12 \\
0.10 \\
0.18 \\
0.15 \\
0.19 \\
0.16 \\
0.16 \\
0.15 \\
0.20 \\
0.19 \\
\text { Averag }\end{array}$ & $\begin{array}{l}0.92 \\
1.13 \\
0.96\end{array}$ & $\begin{array}{l}0.89 \\
0.97 \\
0.95\end{array}$ & $\begin{array}{l}0.88 \\
0.93 \\
0.93 \\
0.95 \\
0.94 \\
1.06 \\
1.14 \\
1.30 \\
1.13 \\
1.07 \\
1.00 \\
1.03\end{array}$ & $\begin{array}{l}\text { Dusdenal ulcer } \\
\text { Duodenal ulcer } \\
\text { Duodenal ulcer } \\
\text { Meningitis } \\
\text { Essential hypertension } \\
\text { Essential hypertension } \\
\text { Lower back pain } \\
\text { Rheumatoid arthritis } \\
\text { Rheumatoid arthritis } \\
\text { Sacro-iliac sprain } \\
\text { Essential hypertension }\end{array}$ \\
\hline
\end{tabular}

p-Acetylaminohippuric acid (X) and diodrast (Y)

\begin{tabular}{|c|c|c|c|c|c|c|c|c|c|c|}
\hline $\begin{array}{l}\text { M.D. } \\
\text { S.A.* } \\
\text { E.B.* } \\
\text { E.B.* } \\
\text { A.S.* }\end{array}$ & $\begin{array}{l}4-7-41 \\
1-7-42 \\
2-4-42 \\
2-6-42 \\
2-11-42\end{array}$ & $\begin{array}{l}6 \\
6 \\
6 \\
6 \\
6\end{array}$ & $\begin{array}{c}692 \\
603 \\
554 \\
503 \\
\operatorname{Re}\end{array}$ & $\begin{array}{l}664 \\
707 \\
516 \\
497\end{array}$ & $\begin{array}{l}0.21 \\
0.20 \\
0.16 \\
0.17 \\
\text { mia }\end{array}$ & \begin{tabular}{l|}
0.21 \\
0.16 \\
0.17 \\
0.17 \\
Averag
\end{tabular} & $\begin{array}{l}1.03 \\
(0.83) \\
1.25 \\
1.01 \\
\text { e } 1.10\end{array}$ & $\begin{array}{l}1.00 \\
(0.79) \\
1.04 \\
1.01 \\
1.02\end{array}$ & $\begin{array}{l}(0.56)^{1} \\
1.22 \\
0.91 \\
0.99 \\
1.04\end{array}$ & $\begin{array}{l}\text { Lung abscess } \\
\text { Deviated nasal septum } \\
\text { Hypertension } \\
\text { Hypertension } \\
\text { Peptic ulcer }\end{array}$ \\
\hline \multicolumn{11}{|c|}{ p-Aminomandelic acid $(\mathrm{X})$ and mannitol $(\mathrm{Y})$} \\
\hline $\begin{array}{l}\text { B.J. } \\
\text { L.Mc. } \\
\text { A.C. } \\
\text { I.N. } \\
\text { M.N. } \\
\text { A.M. } \\
\text { E.F. }\end{array}$ & $\begin{array}{r}10-10-41 \\
11-3-41 \\
11-17-41 \\
11-18-41 \\
2-13-42 \\
2-16-42 \\
2-20-42\end{array}$ & $\begin{array}{r}7 \\
8 \\
6 \\
11 \\
11 \\
15 \\
13\end{array}$ & $\begin{array}{r}78 \\
95 \\
54 \\
81 \\
102 \\
62 \\
69\end{array}$ & $\begin{array}{r}89 \\
99 \\
57 \\
83 \\
105 \\
71 \\
70\end{array}$ & & & & Aver & $\begin{array}{l}0.88 \\
0.96 \\
0.94 \\
0.97 \\
0.97 \\
0.88 \\
0.99 \\
0.94\end{array}$ & $\begin{array}{l}\text { P.I.D. } \\
\text { Grippe } \\
\text { Nephritis } \\
\text { Subdeltoid bursitis } \\
\text { Hypertension } \\
\text { Essential hypertension } \\
\text { Essential hypertension }\end{array}$ \\
\hline
\end{tabular}

${ }^{1}$ Aberrant result possibly attributable in the case of W.O. and S.A. to marked reduction in blood flow, and in the case of C.S. to marked renal hyperemia, in the second series of successive clearances.

* Mannitol clearance used instead of inulin.

$\dagger$ Diodrast and PAH done simultaneously, no successive clearance.

above for the dog, were used in comparing clearances in man. The subjects were convalescent patients on the Third (New York University) Medical Division of Bellevue Hospital. Appropriate priming and sustaining infusions were given as sterile saline solutions of the sodium salts. These solutions were sterilized by ultrafiltration through a Jena bacteriological sintered glass filter, or by heating to $95^{\circ} \mathrm{C}$. for a short period.

p-Hydroxyhippuric acid, m-aminohippuric acid, p-aminohippuric acid, p-acetylaminohippuric acid, diodrast, and hippuran show essentially identical clearances by any of the three methods of comparison. The identity of the $\mathrm{p}$-aminohippuric acid and the diodrast clearances is maintained in 
a variety of diseased states, even when the clearance is reduced by hypertensive disease or chronic glomerular nephritis.

o-Hydroxyhippuric acid shows a clearance significantly lower than that of diodrast, but one which by its magnitude relative to the inulin clearance indicates substantial tubular excretion.

The clearance of p-aminomandelic acid is close to the filtration rate. Although systematic errors of a slight magnitude cannot be ruled out, the deviations between this clearance and that of inulin, especially in long experiments, indicate that the process of excretion of this compound is complex.

It can be said in brief that the human and the dog kidney respond alike to the compounds studied here, as is the case with all compounds so far examined quantitatively except creatinine $(3,25)$.

Determination of $\mathrm{pK}_{\mathrm{a}}^{\prime} \cdot{ }^{\circ}$ As a matter of possible interest in relation to tubular excretion, the dissociation constant of the acidic group of a number of these compounds was determined by titration with $\mathrm{NaOH}$ and measurement of $\left(-\log a_{\mathbf{H}^{+}}\right)$by the glass electrode (see Table VI). In the calculation, $\log \left(\mathrm{a}_{\mathrm{B}^{+}}\right)$is taken as equal to $\log \left(\mathrm{H}^{+}\right)+$ $\log \gamma_{\mathbf{H}^{+}}$, where $-\log \gamma_{\mathbf{H}^{+}}$in dilute solution is approximately 0.05 . Total anion $\left(\mathrm{A}^{-}\right)$is taken as $\left(\mathrm{H}^{+}\right)+\left(\mathrm{B}^{+}\right)$, where $\left(\mathrm{B}^{+}\right)$is the equivalent concentration of base added. The concentration of undissociated acid (HA) is taken as (total acid) $-\left(A^{-}\right)$. With these substitutions,

$$
\mathrm{pK}_{\mathrm{a}}^{\prime}=\mathrm{pH}+\log \frac{\left(\mathrm{A}^{-}\right)}{(\mathrm{HA})}
$$

The $\mathrm{pK}_{\mathrm{a}}^{\prime}$ values are given in Table VI. In the case of $\mathrm{p}$-aminophenylsuccinic acid, the neutralization curve is nearly linear beyond $\left(\mathrm{B}^{+}\right) /(\mathrm{HA})$ $=0.5$, indicating that the two $\mathrm{pK}^{\prime \prime} \mathrm{s}$ do not differ greatly in magnitude, and computation of these values was not attempted.

$\mathrm{pK}_{\mathrm{a}_{2}}^{\prime}$ was determined in the case of o-hydroxyhippuric and $\mathrm{m}$-hydroxyhippuric acids to be 8.40 and 9.26, respectively. The effect of $0.16 \mathrm{M}$ $\mathrm{NaCl}$ was examined in the case of hippuran,

6 We are indebted to Dr. Barbara A. Parker for the determination of the pK's of hippuran, diodrast, and iopax in water and in $0.16 \mathrm{M} \mathrm{NaCl}$, and to $\mathrm{Mr}$. Walter A. Bastedo of the Research Laboratory, Merck and Company, for all other determinations of this value.
TABLE VI

Dissociation constants of substituted hippuric acids, etc. (Determinations made at $23^{\circ}$ to $25^{\circ} \mathrm{C}$.)

$\begin{array}{lcc}\text { Acid } & \begin{array}{c}\text { Initial } \\ \text { total acid } \\ \mathrm{M} \times 10^{3}\end{array} & \text { pK'. } \\ \begin{array}{c}\text { Diodrast } \\ \text { Clearances equal to diodrast: }\end{array} & 3.43 & 2.71 \\ \text { m-Hydroxyhippuric acid } & 32.10 & 3.58 \\ \text { m-Aminohippuric acid } & 29.40 & 4.18 \\ \text { p-Aminohippuric acid } & 19.43 & 3.83 \\ \text { p-Acetylaminohippuric acid } & 14.70 & 3.70 \\ \text { 2-Pyridone-1-acetic acid } & 26.00 & 2.94 \\ \text { Cinnamoylglycine } & 4.60 & 3.57 \\ \text { Hippuran } & 10.06 & 3.63 \\ \text { Clearances less than diodrast: } & 21.70 & 3.56 \\ \text {-Hydroxyhippuric acid } & 7.12 & 2.99 \\ \text { Iopax } & 26.00 & c a .4 .66 \\ \text { p-Aminophenaceturic acid } & 9.12 & 4.62 \\ \text { p-Aminomandelic acid } & & \\ \text { Clearance not determined: } & 13.40 & 3.17 \\ \text { p-Iodomandelic acid } & & \end{array}$

iopax, and diodrast, and showed a reduction in $\mathrm{pK}_{\mathrm{a}}^{\prime}$ of $-0.22,-0.04$, and -0.17 units, respectively. (In the $\mathrm{NaCl}$ solutions, $-\log \gamma_{\mathbf{H}^{+}}$is taken as 0.10 .)

There is no correlation between $\mathrm{pK}_{\mathrm{a}}^{\prime}$ and the manner in which these substances are handled by the kidney. Recognizing that phenol red $\left(\mathrm{pK}^{\prime}=7.9\right)$ is also abundantly excreted by the renal tubules in man (8), it appears that within wide limits the acidic strength has little bearing on whether a substance will or will not be handled in this manner.

Determination of filterable p-aminohippurate in plasma. Attempts were made to measure the ultrafilterable p-aminohippurate in dog and human plasma by the collodion bag method previously used in this laboratory in connection with studies of phenol red, diodrast, etc. (9), but we were unable to obtain reproducible results. (Shannon has adduced evidence that constituents of parlodion react with aryl amines, possibly by way of nitration, with the consequence that dialysis experiments with such compounds in parlodion bags give erratic results. Personal com.) We then decided to obtain the necessary data directly in man by following the rate of excretion on a falling plasma curve, with the plasma concentration always above the level required to effect saturation of the tubules. It will be seen from the following equation

$$
\mathrm{Tm}_{\mathrm{PAB}}=\mathrm{U}_{\mathbf{P A B}} \mathrm{V}-\mathrm{C}_{\mathrm{IN}} \mathrm{P}_{\mathbf{P A B}} \mathrm{b}
$$

that if $\mathrm{U}_{\mathrm{PAB}} \mathrm{V}$ is plotted against $\mathrm{C}_{\mathrm{IN}} \mathrm{P}_{\mathbf{P A H}}$, the points should fall in a straight line (assuming $b$ 
is constant), the slope of which will be equal to $b$, the percentage of $\mathrm{PAH}$ in the plasma which is ultrafilterable (here $b$ corresponds to $\mathrm{FW}$ in the equation of Smith, Goldring, and Chasis (2)), and that the intercept on the $\mathrm{U}_{\mathrm{PAH}} \mathrm{V}$ axis will be equal to $\operatorname{Tm}_{\mathbf{P A B}}$. In the above calculations, the best straight line was fitted by the method of least squares to the data obtained from 8 successive clearance periods, with the plasma concentration falling roughly in the range from 100 down to 25 mgm. per cent. The average value thus determined in 11 subjects was 0.83 . This value is close enough to unity so that changes in $b$, related to changes in $P_{\mathbf{P A H}}$ in the range of 25 and 100 mgm. per cent, can be neglected in the calculations of $\mathrm{Tm}_{\mathrm{PAH}}$, i.e., the average value of 0.83 can be used with little error in the calculation, as in the case of diodrast. Unfortunately, we were unable to examine the effect of plasma protein concentration on $b$, but for practical purposes or except in special studies we believe that neglect of this factor will result in a negligible error in $\mathrm{Tm}$ calculation.

Conjugation of the p-amino group. The question of possible conjugation on the p-amino group is important since at least one conjugate, $\mathrm{p}$-acetylaminohippuric acid, is also excreted by the tubules; conjugation would occlude the p-amino position during analysis for $\mathrm{p}$-aminohippuric acid and any unanalyzed conjugate derivative present in the plasma during the determination of $\mathrm{p}$ aminohippuric acid $\mathrm{Tm}$ might contribute to saturation of the tubules and thus reduce the $T m$ value of the latter. Conjugation would probably be of no importance with respect to p-aminohippuric acid clearance determination since there is no reason to believe that the conjugate derivative would interfere with the excretion of p-aminohippuric at low plasma levels, while it would not appear in the analytical method for the latter compound as applied to either plasma or urine.

Twenty-six samples of plasma and 27 samples of urine, collected during routine determination of p-aminohippuric acid $\mathrm{Tm}$ in man (10), analyzed by the volumetric flask method (see Methods), showed no significant amount of conjugated derivative, the average ratio of free to total acid being 1.00 for both the blood and urine series. (The plasma concentration ranged from 31 to 89 mgm. per cent, in some cases increasing, in others decreasing, at moderate rates during the Tm determination.) It is therefore concluded that during the infusion of $\mathrm{p}$-aminohippuric acid in the concentrations used for $\mathrm{Tm}$ determination, the absolute quantity of material conjugated in the p-position is so small as to be negligible, relative to the total quantity undergoing excretion.

However, when a single dose of 1.0 gram of sodium $\mathrm{p}$-aminohippurate was given orally to a normal subject and the total urine collected for 6 hours, 65 per cent of the administered material was conjugated. In 2 subjects during a p-aminohippuric acid clearance determination, the extent of conjugation in 3 urine samples each was 9.9, $12.6,12.8$, and $7.6,9.1,11.5$ per cent respectively. It is clear that when the body is not presented with an overwhelming quantity, the proportion conjugated is significant. This observation is in line with the known conjugation of p-aminobenzoic acid $(11,12)$ and of sulfanilamide (11, $13,14)$ in man.

Examination of dog urine by open tube hydrolysis and corrected for blank excretion during a preliminary period after the intravenous administration of $200 \mathrm{mgm}$., showed no appreciable conjugation. It thus appears that conjugation of $\mathrm{p}$-aminohippuric acid follows the behavior of $\mathrm{p}$ aminobenzoic acid (11) and of sulfanilamide (11, 14 ), in that conjugation of the p-amino group does not occur in the dog.

Adverse physiological reactions. Five-tenths of a gram per $\mathrm{kgm}$. of sodium p-aminohippurate can be given to dogs intravenously by syringe over a period of 5 to 10 minutes with no adverse reaction. Repeated determinations of clearances and Tm values have revealed no disturbance of renal function in dogs examined over several months. Administration of large quantities to man for $\mathrm{Tm}$ determination is described elsewhere (10).

Use of p-aminohippuric acid for the evaluation of effective renal blood flow and tubular excretory mass. In view of the following facts, sodium $\mathrm{p}$ aminohippurate (PAH) appears to be suitable for the evaluation of those aspects of renal function which have hitherto been evaluated by the use of diodrast :

(a) At low plasma levels, the clearance is identical with that of diodrast, and hence equally valid as a measure of the effective renal plasma flow. 
(b) The chemical determination is extremely simple, using reagents available in all clinical laboratories, the method as here described giving quantitative and easily reproducible values from suitable plasma filtrates and urine dilutions.

(c) The endogenous plasma and urine blanks are negligibly small.

(d) It does not penetrate the human red cell in vivo, even on prolonged infusion, and hence any possible error attributable to extraction from the blood cell during passage through the kidneys is obviated.

(e) It is non-toxic, and can be used for the evaluation of the tubular excretory mass by the saturation method.

(f) It is less extensively bound to plasma proteins than is diodrast ( $F W=0.83$ as compared with 0.73 for the latter), and hence errors involved in the estimation of the percentage of free and filterable material in the plasma are of less practical significance.

A comparison of the maximal rate of tubular excretion of $\mathrm{p}$-aminohippuric acid $\left(\mathrm{Tm}_{\mathrm{PAH}}\right)$ with the maximal rate of tubular excretion of diodrast $\left(\mathrm{Tm}_{\mathrm{D}}\right)$ in the normal and diseased human kidney will be presented in a following paper.?

\section{SUMMARY}

Methods are described for the determination of hydroxy- and amino-substituted hippuric acid derivatives in urine and in protein-free filtrates of plasma. The chromogenic blanks, as given by these methods, have been determined in proteinfree filtrates from plasma and red cells in dog and man, and recoveries of the compounds from plasma and red cells have been determined in both species. The in vivo distribution between red cells and plasma of most of the compounds is reported for both species. It is noteworthy that

${ }^{7} \mathrm{p}$-Aminohippuric acid $\mathrm{Tm}$ has been measured in 3 dogs $(F W=0.87$ to 0.92$)$, the ratio of this value over diodrast $\mathrm{Tm}$ determined within an interval of several weeks and calculated on a molar basis being 1.00, 1.10, and 0.93 . Less reliable data indicate that the molar ratio of p-hydroxyhippuric acid $\mathrm{Tm}(\mathrm{FW}=0.77$ to 0.815$)$ to diodrast $\mathrm{Tm}$ in 3 dogs was $1.18,0.59$, and 0.84 . From comparative data on man (10), it is clear that a fixed molar value of $\mathrm{Tm}$ for different compounds is not to be anticipated in different species, although the molar ratio in one species appears to be fairly constant. the human red cell is much less permeable in vivo to all the compounds studied than is the dog red cell, and that in no case, even after prolonged infusion, does the cell/plasma distribution ratio (per unit of water) approach unity.

The plasma renal clearances have been compared in the dog by $(a)$ the ratio of the successive clearances in two closely following sets of clearance determinations, each comprised of three clearance periods; $(b)$ the ratio of successive filtration fractions relative to creatinine, inulin, or mannitol, in these two sets of successive clearance periods; and (c) the ratio of the simultaneous clearances.

The clearances of (1) m-hydroxyhippuric acid, (2) p-hydroxyhippuric acid, (3) m-aminohippuric acid, (4) p-aminohippuric acid, (5) p-acetylaminohippuric acid, (6) 2-pyridone-1-acetic acid, (7) cinnamoylglycine, (8) diodrast (3-5 di-iodo4-pyridone-1-acetic acid), and (9) hippuran (oiodohippuric acid) are essentially identical in the dog. Only compounds $2,3,4,5$, and 9 have been examined in man, and here too the clearances are identical. This fact conforms with the premise that the limiting circumstance in the tubular excretion of diodrast (or of any one of these substances) is the available blood flow to the renal tubules (approximating the total renal blood flow), rather than a limitation in the tubular excretory mechanism.

The fact that the clearances of p-aminohippuric acid, which does not penetrate the red cell in vivo in man, and of diodrast which does penetrate, are identical under all conditions, including renal hyperemia, indicates that no significant quantity of diodrast moves out of the red cell during the renal passage of the blood.

The clearances of o-hydroxyhippuric acid, paminophenylaceturic acid, iopax, and cinnamoylglycine in the dog, although greater than the creatinine clearance, are significantly less than the clearances of diodrast and substituted hippuric acids. The o-hydroxyhippuric acid clearance is similarly lower than the diodrast clearance in man. This deficiency in clearance is presumably related to limitations in the tubular excretory mechanism.

The p-aminobenzoic acid clearance in the dog is initially less than that of creatinine, indicating tubular reabsorption (plasma binding has not been studied) but on prolonged infusion, the clearance 
rises to high values indicating conjugation to $\mathrm{p}$ aminohippuric acid.

The p-aminophenylsuccinic acid clearance in the dog is greater than the creatinine clearance and increases on prolonged infusion, indicating metabolism to some substance with a high clearance value.

The clearance of p-aminomandelic acid appears to be slightly above that of creatinine, in the dog, and slightly below that of inulin in man. The discrepancies, however, are only slightly. beyond the limits of systematic errors in one or the other analytical procedures.

$\mathrm{p}$-Aminohippuric acid is conjugated in the $\mathrm{p}$ position in man but not in the dog. The presence of conjugated material does not interfere with the use of this compound for clearance determination, since it does not enter into the analytical method for either plasma or urine. The absolute quantity conjugated during p-aminohippuric Tm determination is so small as to be negligible, and offers no difficulty in the use of $\mathrm{p}$-aminohippuric acid in the saturation method for evaluating the total quantity to tubular excretory tissue.

Since the analytical method for $\mathrm{p}$-aminohippuric acid is much simpler than that for diodrast, and since the former compound does not penetrate the red cell in man and is less extensively bound to plasma protein than is diodrast, it is recommended for the evaluation of the effective renal blood flow and tubular excretory mass. (It must of course be administered to man as an approximately neutral, dilute, and sterile solution of the sodium salt.)

\section{METHODS}

Manipulative. It is our custom to use $2 \mathrm{ml}$. or larger samples of plasma for precipitation, and $2 \mathrm{ml}$. samples of urine for dilution. A $1.0 \mathrm{ml}$. pipette is never used except for the addition of reagents, the exact volume of which is not important. Dilutions of urines and standards are preferably made with pipettes and volumetric flasks rather than by the addition of water from a burette. We prefer heavy tipped, pear-shaped blowout pipettes, made to order in all sizes by E. Machlett and Sons, New York; if ordinary pipettes are used, they should be selected by weighing expelled water or mercury. Pipettes are rinsed with water, soaked for 2 to 18 hours in cleaning fluid, rinsed in an automatic pipette washer, rinsed with alcohol, and dried at room temperature over night.

Blood samples are centrifuged at once and the plasma removed and precipitated between successive samples.
In clinical work, the separated plasma is returned to the analytical laboratory for precipitation, but 2 or $5 \mathrm{ml}$. of urine are routinely diluted to 50 in a volumetric flask and transferred to $25 \times 200 \mathrm{~mm}$. tubes, which are stoppered and labelled at the bedside, further dilutions being made in the analytical laboratory.

In dog studies, it is convenient to introduce a retention needle (No. 19, 21/2 in., medium bevel, made to order by Becton, Dickinson and Co.) bearing a locking, blunt tipped stylet, into the jugular vein and to leave it in place throughout clearance determination. Clotting can generally be prevented by wetting the stylet with saturated potassium oxalate solution each time it is removed. Similar retention needles are useful for repeated arterial or venous blood samples in man.

Constant intravenous infusions are given into the ear vein or posterior tibial vein in the dog by means of a mercury gravity pump comprised of a 125 or $250 \mathrm{ml}$. side-arm filter flask, a standardized capillary tip in the rubber stopper of this flask, a $250 \mathrm{ml}$. levelling bulb, and rubber tubing. The capillary tip may be made by grinding back a fairly thick broken pipette tip, fused in a flame until one can barely blow a jet of air through the lumen. It should deliver from 0.5 to $1.0 \mathrm{ml}$. per minute of mercury when the latter is fed from the mercury reservoir fixed at a convenient height. One hundred to $150 \mathrm{ml}$. of infusion are placed in the side-arm flask after the end of infusion tubing has been fastened at a level slightly above the top of the flask by a small piece of adhesive tape. Mercury is then added until the level of fluid is well above the top of the side arm. The pendant portion of the infusion tubing is examined to be sure it is free of air bubbles. The stopper and capillary tip attached to the mercury reservoir are hung in a bracket above the mercury level in the reservoir. All that is required to start the infusion is to lift the stopper from its bracket and to insert it tightly into the mouth of the flask. The infusion tubing between the side-arm flask and the vein should be of small caliber ( $1 / 16$ in. internal diameter), in order to reduce the weight on the needle, but a section of larger gum tubing which can be punctured in order to introduce priming injections, should be inserted close to the side-arm flask. Introduced slowly, such priming injections are prevented by pressure from moving backwards into the flask. All rubber tubing bearing mercury under pressure must be tightly wired to glass joints.

Dog infusion needles are made by sweating a brass collar 2 to $3 \mathrm{~mm}$. over-all diameter and 8 to $10 \mathrm{~mm}$. long, and ringed at several points, on to No. 19 or 20 , $11 / 2$ inch medium to short bevel hypodermic needles after cutting off the butts. The small infusion tubing must have a round lumen of such a size as to slip on to this collar readily. In starting the infusion, the needle is wet inside with a drop of oxalate solution and inserted into the shaved ear vein. With the infusion running, the infusion tubing is slipped firmly on to the needle and, while an assistant holds the needle in place, the tubing is tied over the collar with strong thread. An alternative method is to insert a small piece of glass tubing in the 
infusion tubing, $1 / 2$ to $3 / 4$ of an inch from the end, which may now be firmly and permanently placed on the infusion needle. Successful puncture of a vein is revealed by a flush of blood into the glass segment when the infusion tubing, momentarily occluded, is squeezed and released.

After venipuncture is completed, the needle is fastened in the vein by placing a small celluloid strip on the under side of the ear and slipping a paper clip over the shaft of the needle and the celluloid strip. The ear is then folded on its long axis, and wrapped lightly with $1 / 2$ inch adhesive tape, the infusion tubing being similarly fastened to the ear after looping once to protect the end from strain. By this method, infusions can be given to a dog in any position for several hours with rare accidental interruptions. The same vein may be used repeatedly. Clotting in the needle may occur if a rise in venous pressure pushes blood back against the infusion stream, but it is quickly detected by the escape of a fine stream of air bubbles if a water seal is floated around the rubber stopper closing the side-arm flask.

Intravenous infusions in man are given aseptically into an arm vein, using a 4-inch tunnel clamp to control the rate, the latter being measured by counting the drops of a Murphy drip.

In dogs (females), the bladder is catheterized with a No. 14 or 16 flexible catheter with 4 holes. In man, appropriate male or female catheters are used. It is convenient to rinse the bladder of the dog by running in $10 \mathrm{ml}$. of water from a Folin-Wu $15 \mathrm{ml}$. pipette; by expelling the contents into the pipette again a very good idea can be obtained of the efficiency of emptying. In man, rinsing is effected by injecting and withdrawing sterile saline with a sterile syringe.

Precipitation. In earlier work (see footnotes to Table I), we used a conventional $1: 5$ or $1: 10$ trichloroacetic acid filtrate, prepared by adding 3 or 8 volumes, respectively, of water to 1 volume of plasma or hemolyzed cell suspension, followed by 1 volume of 20 per cent trichloroacetic acid; or a tungstate filtrate prepared by adding 2 or 7 volumes of water, respectively, to 1 volume of plasma plus 1 volume of 10 per cent $\mathrm{Na}_{2} \mathrm{WO}_{4}$, followed by 1 volume of $0.66 \mathrm{~N} \mathrm{H}_{2} \mathrm{SO}_{4}$. These filtrates suffered the disadvantage that they did not serve equally well for all determinations, and consequently the cadmium sulfate filtrate was later adopted. This filtrate has the advantage of supplying a dry, protein-free solution, useful for many purposes.

Cadmium sulfate- $\mathrm{NaOH}$ precipitation (adapted from Fujita and Iwatake (15).

(a) 34.667 grams $3 \mathrm{CdSO}_{4} .8 \mathrm{H}_{2} \mathrm{O}$ plus $169.3 \mathrm{ml} . \mathrm{N}$ $\mathrm{H}_{2} \mathrm{SO}$, made up to $1000 \mathrm{ml}$.

(b) $1.1 \mathrm{~N} \mathrm{NaOH}$.

Plasma (1:15). To 1 volume of plasma in a $125 \mathrm{ml}$. Erlenmeyer flask, add 10 volumes of water and 3 volumes of $(a)$ and $\mathrm{mix}$; then add 1 volume of $(b)$, close with a rubber stopper and shake well. After 10 minutes, centrifuge and decant the supernatant fluid through filter paper or washed cotton. (If creatinine is to be determined in this filtrate, washed cotton should be used or the filter paper should be tested for a chromogenic blank by the additive method.)

When used for the determination of creatinine, a trace of cadmium may precipitate from this filtrate on the addition of alkaline picrate solution; this does not, however, significantly reduce the alkalinity or retard color development, and recovery of added creatinine, above the endogenous chromogen as determined by addition of creatinine to plasma filtrates, is 100 per cent. It has been our custom to filter through washed cotton (filter paper should not be used) or to centrifuge the alkaline picrate solution during the 10-minute interval required for color development in order to remove the precipitated cadmium.

The above reagents may be used to prepare a $1: 5$ filtrate by using 1 volume of plasma, 3 volumes of $(a)$, and 1 volume of $(b)$. In some instances, however, recoveries on a 1:5 filtrate are less than 100 per cent.

Cells. Red blood cells are centrifuged at 3000 r.p.m. for at least 30 minutes and the supernatant plasma carefully removed by a capillary pipette. (In the calculation it is assumed that the cell mass contains $\mathbf{5}$ per cent interstitial plasma.) About 1 volume of distilled water, measured from a burette, is stirred into the cells to permit decantation, and the mixture is transferred with minimal washing into a graduated cylinder, and the volume made up to approximately 2.5 times the volume of cells taken, as determined by the difference between total volume in the cylinder and the volume of water added. The mixture is then well stirred until hemolysis is complete. Without hemolysis, recovery of intracellular constituents may be low. An aliquot of this hemolyzed suspension is transferred with a wash-out pipette into a $125 \mathrm{ml}$. Erlenmeyer flask and precipitated by the addition of 3 volumes of $(a)$ and 1 volume of $(b)$ as above, with special care for mixing and thorough precipitation by shaking.

Blanks are determined by adding known quantities of substrate to appropriate plasma or cell filtrates.

Urines are diluted as nearly as possible to a $U / P$ ratio of 1.0, and treated like plasma filtrates.

Colorimetric determinations. General. Standard colorimeter curves are plotted on semi-logarithmic paper (Keuffel and Esser No. 358-51) and labelled in respect to date, reagent number, etc. Plastic tubes can be used in the p-amino coupling, but glass tubes are generally preferable for alkaline reagents such as alkaline picrate.

Since it is difficult to maintain a large stock of perfectly matched Evelyn colorimeter tubes, it is advantageous to use a pair of matched tubes, both marked for position in the colorimeter, one for the reagent blank and one for the unknowns. Hence it is desirable to have a total volume of at least $12 \mathrm{ml}$. in the coupling reaction in order to have enough solution for rinsing. The adherent drop remaining after emptying should be removed by inverting the colorimeter tube on clean tissue, and the optically exposed portion of the tube should be wiped clean with tissue between each sample. The tube should always be placed in the colorimeter in the same position.

The 100 per cent transmission setting of the galvanometer should be made with a complete reagent blank 
contained in the second matched colorimeter tube, and this 100 setting (or the corresponding air reading if the blank is stable) checked frequently during a series of determinations. The zero setting of the galvanometer should be checked daily.

In no case should a plasma blank be used for the 100 setting of the galvanometer. If a plasma blank is present, it should be determined additively, as described in the text, and applied to the recovery determination as well as to the unknowns. Otherwise large errors may be introduced by a deficient recovery in plasma precipitation obscured by a compensating plasma blank.

$o$ - and $m$-Hydroxyhippuric acids. The following method is a modification of that of Gibbs (16) :

(a) Veronal buffer: a 2 per cent sodium veronal solution adjusted to $\mathrm{pH} 9.1$ with $\mathrm{HCl}$ and $\mathrm{NaOH}$.

(b) 2,6-Dichloroquinonechloroimide solution, $25 \mathrm{mgm}$. per cent in absolute alcohol. This solution should be freshly prepared and centrifuged if not clear. It will keep for only a few hours.

To $10 \mathrm{ml}$. of plasma filtrate or diluted urine, add 2 $\mathrm{ml}$. of $(a)$ and $2 \mathrm{ml}$. of $(b)$, shaking the mixture after the addition of each reagent. Solution $(b)$ should be added immediately after $(a)$, since hydroxy acids deteriorate rapidly in alkaline solution. A blank of 10 $\mathrm{ml}$. of water is treated in the same manner and used for the 100 setting of the colorimeter. Maximal absorption of the indophenol is at $600 \mathrm{mu}$. but a $635 \mathrm{mu}$. Rubicon filter is satisfactory. A series of standards prepared from $10 \mathrm{ml}$. samples of 0.05 to $0.30 \mathrm{mgm}$. per cent solutions of o-hydroxyhippuric acid, or 0.1 to $0.6 \mathrm{mgm}$. per cent solutions of $\mathrm{m}$-hydroxyhippuric acid should be prepared each time, using the same alcoholic chloroimide solution as is used in the unknowns. Maximal color development is attained in 30 minutes with o-hydroxyhippuric acid and in 60 minutes with $\mathrm{m}$-hydroxyhippuric acid.

When increasing amounts of $\mathrm{m}$-hydroxyhippuric acid are added to aliquots of the same plasma filtrate, and the resulting apparent concentrations are plotted against the concentrations in equivalent aqueous dilutions, the slope of the curve is 0.82 , indicating that there is present in the filtrate something that uniformly inhibits color development. This inhibition has been taken in account in the calculation of both plasma recoveries and clearances of this compound. In addition, there is a small, positive intercept, indicating a true blank.

$p$-Hydroxyhippuric acid. This method is based on that of Arnow (17), and gives somewhat greater color than does Arnow's procedure.

(a) 20 per cent $\mathrm{HgSO}_{4}$ in $5 \mathrm{~N} \mathrm{H}_{2} \mathrm{SO}_{4}$

(b) 0.2 per cent $\mathrm{NaNO}_{2}$ solution

To $5 \mathrm{ml}$. of plasma filtrate or diluted urine in a 25 by $200 \mathrm{~mm}$. heavy-walled Pyrex tube, add $7 \mathrm{ml}$. of a fresh mixture containing 3 parts of $(a)$ and 4 parts of $(b)$. Close the tube with a glass tear and immerse in boiling water for $4 \frac{1}{2}$ minutes. Cool in water at room temperature, shake and read on the colorimeter with a $490 \mathrm{mu}$. filter, using a blank prepared from $5 \mathrm{ml}$. of water and $7 \mathrm{ml}$. of reagent and treated as above for the 100 setting.
The color is stable for at least 1 hour. Although the standard curve is fairly reproducible, it is desirable because of the short heating period to include standards ( $5 \mathrm{ml}$. each of solutions containing 0.4 to $4.0 \mathrm{mgm}$. per cent) with each lot of unknowns.

$m$ - and $p$-Aminohippuric acids, p-aminophenaceturic acid, p-aminophenylsuccinnic acid, p-aminobenzoic acid, and p-aminomandelic acid. All these compounds couple satisfactorily in Bratton and Marshall's (18) method for sulfanilamide. The slight modifications presented here have, however, proved to be practically advantageous.

(a) $1.2 \mathrm{~N} \mathrm{HCl}$

(b) $100 \mathrm{mgm}$. per cent $\mathrm{NaNO}_{2}$

(c) $500 \mathrm{mgm}$. per cent ammonium sulfamate

(d) $100 \mathrm{mgm}$. per cent $\mathrm{N}$-(1-naphyl) ethylenediamine dihydrochloride

To $10 \mathrm{ml}$. of plasma filtrate or diluted urine add $2 \mathrm{ml}$. of $(a)$ and $1 \mathrm{ml}$. of $(b)$, and $\mathrm{mix}$ the solution; not before 3 minutes and not after 5 minutes, add $1 \mathrm{ml}$. of (c) and $\mathrm{mix}$; between 3 and 5 minutes later, add $1 \mathrm{ml}$. of (d) and mix. A blank made of $10 \mathrm{ml}$. of water treated in the same manner is used to set the colorimeter at 100 . Solutions are read at any time after 10 minutes, using a $540 \mathrm{mu}$. filter.

With p-aminohippuric acid, color development is maxi$\mathrm{mal}$ in $\mathbf{5}$ minutes, and the color is quite stable.

A standard curve is set up with $10 \mathrm{ml}$. samples of solution ranging in concentration from 0.02 to $0.25 \mathrm{mgm}$. per cent of free p-aminohippuric acid, the acid being dried if necessary at $90^{\circ} \mathrm{C}$. overnight before preparation of the stock solution. The sodium salt is too hygroscopic to use as a standard. The standard curve is quite reproducible and needs to be rechecked only on rare occasions. A separate curve, however, should be prepared for each colorimeter.

The $\mathrm{NaNO}_{2}$ must be prepared fresh every 3 days, the ammonium sulfamate every 2 weeks; the coupling reagent $(d)$ lasts indefinitely if kept in a dark bottle and in the refrigerator. p-Aminohippuric acid is stable in plasma filtrates and urines if kept in the ice box.

(Bratton and Marshall (18) recommend a trichloroacetic acid filtrate for sulfanilamide, but we have found this filtrate to be quite unreliable for p-aminohippuric acid. Although perfect recoveries may be obtained, the coupling reaction frequently and inexplicably fails to go forward quantitatively or reproducibly. No such difficulty has ever been encountered with the cadmium sulfate filtrate, which gives excellent checks in duplicate determinations on independent precipitations.)

The chromogenic power of various compounds when corrected for molecular weight (but not specially dried) and the time required for maximal color development are as follows: p-aminohippuric, 100 per cent $<10$ minutes; m-aminohippuric, 96 per cent, 30 minutes; paminobenzoic, 97 per cent, 20 minutes; p-aminomandelic, 90 per cent, 60 minutes; p-aminophenylsuccinic, 75 per cent, 90 minutes; p-aminophenaceturic, 83 per cent, 5 hours. In all cases, the color is quite stable.

p-Acetaminohippuric acid. Hydrolysis to p-aminohippuric acid is carried out by the addition of $2 \mathrm{ml}$. of 
$1.2 \mathrm{~N} \mathrm{HCl}$ to $10 \mathrm{ml}$. of filtrate or diluted urine, contained in graduated narrow test tubes or Folin sugar tubes. After thorough mixing, the tubes are closed with glass tears and heated in a boiling water bath for 1 hour. The tubes are then cooled and the volume made up to $12.5 \mathrm{ml}$. and the p-aminohippuric acid content determined as above. If read against the standard p-aminohippuric acid curve, prepared as above, correction must be made for the increased total volume after the addition of coupling reagents, i.e., the result must be multiplied by $12.5+5 / 10+5$ or 1.17 .

Recoveries by this method are imperfect, some of the chromogen being destroyed, perhaps by oxidation. (Similar imperfect recovery after hydrolysis of p-acetylaminobenzoic acid is reported by Doisy and Westerfeld (19).) It is therefore preferable to prepare a standard curve by treating knowns in the manner of unknowns. Since the extent of loss appears to be fairly uniform in filtrates and urines, no large error is introduced in clearance determination.

Conjugated p-aminohippuric acid. Since absolute recoveries were essential in answering the question of possible conjugation in the body, the following method, which appears to be wholly satisfactory, was developed later in the investigation: $2 \mathrm{ml}$. of $10 \mathrm{~N} \mathrm{HCl}$ are added to suitable aliquots of plasma filtrate or diluted urine in $50 \mathrm{ml}$. volumetric flasks, made up to volume and mixed. The solution in the neck is covered with about $1 \mathrm{ml}$. of toluene. The flasks are placed in an oven at approximately $96^{\circ} \mathrm{C}$. for $3 \frac{1}{2}$ hours. After cooling, $10 \mathrm{ml}$. of this solution are taken for analysis by the p-aminohippuric acid method. p-Aminohippuric acid when treated in this manner gives 100 per cent recovery, as compared with analysis of an unheated solution. For this reason, we believe that recovery of conjugated material is essentially complete. (A presumptively pure sample of p-acetylaminosulfanilamide gave 95 per cent recovery by this method.)

Diodrast was determined by Alpert's method (20). In man, recovery in the $1: 15$ cadmium sulfate filtrate averaged 94 per cent (cf. Alpert's paper) but in dog recovery averaged so close to 100 per cent that no correction has been made. Hippuran and iopax were determined by the fusion method (2). Inulin was used in quantities sufficient to give plasma concentrations of 80 to $150 \mathrm{mgm}$. per cent and determined by the macromethod (2) on a $\mathrm{ZnSO}_{4}-\mathrm{NaOH}$ filtrate (21). Mannitol was determined by the method of Smith, Finkelstein, and Smith (21).

2-Pyridone-1-acetic acid and cinnamoylglycine were determined in cadmium sulfate filtrates by a Hilger medium two-beam ultraviolet spectrograph with Spekker attachment, using standard procedures. We are indebted to Dr. Rudolph Naumann for these determinations.

\section{BIBLIOGRAPHY}

1. Elsom, K. A., Bott, P. A., and Shiels, E. H., On the excretion of skiodan, diodrast and hippuran by the dog. Am. J. Physiol., 1936, 115, 548.
2. Smith, H. W., Goldring, W., and Chasis, H., The measurement of the tubular excretory mass, cffective blood flow and filtration rate in the normal human kidney. J. Clin. Invest., 1938, 17, 263.

3. Shannon, J. A., Renal tubular excretion. Physiol. Rev., 1939, 19, 63.

4. White, H. L., Observations on the behavior of diodrast in the dog. Am. J. Physiol., 1940, 130, 454.

5. Chasis, H., Ranges, H. A., Goldring, W., and Smith, H. W., The control of renal blood flow and glomerular filtration in normal man. J. Clin. Invest., 1938, 17, 683.

6. Smith, W. W., and Ranges, H. A., Renal clearances of iopax, neoiopax and skiodan in man. Am. J. Physiol., 1938, 123, 720.

7. Smith, H. W., Note on the interpretation of clearance methods in the diseased kidney. J. Clin. Invest., 1941, 20, 631.

8. Goldring, W., Clarke, R. W., and Smith, H. W., The phenol red clearance in normal man. J. Clin. Invest., 1936, 15, 221.

9. Smith, W. W., and Smith, H. W., Protein binding of phenol red, diodrast, and other substances in plasma. J. Biol. Chem., 1938, 124, 107.

10. Chasis, H., Redish, J., Goldring, W., Smith, H. W., and Ranges, H. A., The use of sodium p-aminohippurate for the functional evaluation of the human kidney. J. Clin. Invest., In press.

11. Bernhard, K., Uber die Herkunft der Essigsäure bei den Acetylierungen in vivo. I. Die Acetylierung von Sulfanilamid und p-Amino-benzolsäure bei gleichzeitigen Gaben von Deutero-essigsäure. Z. f. Physiol. Chem., 1940, 267, 91.

12. Strauss, E., Lowell, F. C., and Finland, M., Observations on the inhibition of sulfonamide action by para-aminobenzoic acid. J. Clin. Invest., 1941, 20, 189.

13. Kirch, E. R., and Bergeim, O., Determination of $p$ aminobenzoic acid. J. Biol. Chem., 1943, 148, 445.

14. Marshall, E. K., Jr., Cutting, W. C., and Emerson, $\mathrm{K}$., Jr., Acetylation of para-aminobenzenesulfonamide in the animal organism. Science, 1937, 85, 202.

15. Fujita, A., and Iwatake, D., Bestimmung des echten Blutzuckers ohne Hefe. Biochem. Ztschr., 1931, 242, 43.

16. Gibbs, H. D., Phenol tests. III. The indophenol test. J. Biol. Chem., 1927, 72, 649.

17. Arnow, L. E., Colorimetric determination of the components of 3,4-dihydroxyphenylalanine-tyrosine mixtures. J. Biol. Chem., 1937, 118, 531.

18. Bratton, A. C., and Marshall, E. K., Jr., A new coupling component for sulfanilamide determination. J. Biol .Chem., 1939, 128, 537.

19. Doisy, E. A., Jr., and Westerfeld, W. W., The relationship of acetoin to metabolic acetylations. J. Biol. Chem., 1943, 149, 229.

20. Alpert, L. K., A rapid method for the determination of diodrast-iodine in blood and urine. Bull. Johns Hopkins Hosp., 1941, 68, 522. 
21. Smith, W. W., Finkelstein, N., and Smith, H. W., Renal excretion of hexitols (sorbitol, mannitol, and dulcitol) and their derivatives (sorbitan, isomannide, and sorbide) and of endogenous creatininelike chromogen in dog and man. J. Biol. Chem., 1940, 135, 231.

22. Finkelstein, N., Aliminosa, L. M., and Smith, H. W., The renal clearances of hippuric acid and pyridone derivatives. Am. J. Physiol., 1941, 133, 276.
23. Corcoran, A. C., Smith, H. W., and Page, I. H., The removal of diodrast from blood by the dog's explanted kidney. Am. J. Physiol., 1941, 134, 333.

24. Phillips, R., Emerson, K., Jr., Dole, V. P., Hamilton, P., Archibald, R. M., and Van Slyke, D. D., Personal communication.

25. Shannon, J. A.. The renal excretion of creatinine in man. J. Clin. Invest., 1935, 14, 403. 